\title{
Article
}

\section{Temporal Evolution of Heavy-Ion Spectra in Solar Energetic Particle Events}

Doran, Donald Joseph, Dalla, Silvia and Zelina, Peter

Available at http://clok.uclan.ac.uk/28457/

Doran, Donald Joseph, Dalla, Silvia ORCID: 0000-0002-7837-5780 and Zelina, Peter (2019) Temporal Evolution of Heavy-Ion Spectra in Solar Energetic Particle Events. Solar Physics, 294 (5). ISSN 0038-0938

It is advisable to refer to the publisher's version if you intend to cite from the work. http://dx.doi.org/10.1007/S11207-019-1431-9

For more information about UCLan's research in this area go to

http://www.uclan.ac.uk/researchgroups/ and search for <name of research Group>.

For information about Research generally at UCLan please go to http://www.uclan.ac.uk/research/

All outputs in CLoK are protected by Intellectual Property Rights law, including Copyright law. Copyright, IPR and Moral Rights for the works on this site are retained by the individual authors and/or other copyright owners. Terms and conditions for use of this material are defined in the policies page. 


\title{
Temporal Evolution of Heavy-Ion Spectra in Solar Energetic Particle Events
}

\author{
Donald J. Doran ${ }^{1} \cdot$ Silvia Dalla ${ }^{1} \cdot$ Peter \\ Zelina $^{1,2}$
}

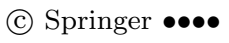

\begin{abstract}
Solar energetic particles (SEPs) are released into the heliosphere by solar flares and coronal mass ejections (CMEs). They are mostly protons, with smaller amounts of heavy ions from helium to iron, and lesser amounts of heavier species than iron. The spectra of heavy ions has been previously studied mostly by using the fluence of the particles in an event integrated spectrum in a small number of spectral snapshots. In this article, we analyze the temporal evolution of the heavy ion spectra using two large SEP events (27 January 2012 and 7 January 2014) from the Solar TErrestrial Relations Observatory (STEREO) era using Advanced Composition Explorer (ACE) Solar Isotope Spectrometer (SIS) and Cosmic Ray Isotope Spectrometer (ULEIS), Energetic Particles: Acceleration, Composition and Transport (EPACT) onboard Wind, and the STEREO-A (Ahead) and -B (Behind) Low-Energy Telescope (LET) and Suprathermal Ion Telescope (SIT) instruments, taking a large number of snapshots covering the temporal evolution of the event. We find large differences in the spectra of the ions after the main flux enhancement in terms of the grouping of similar species, but also in terms of the location of the instruments. Although it is somewhat less noticeable than in the case of the temporal evolution of protons (Doran and Dalla, 2016), we observe a wave-like pattern traveling through the heavy ion spectra from the highest energies to the lowest, creating an "arch" structure that later straightens into a power law after 18 to 24 hours.
\end{abstract}

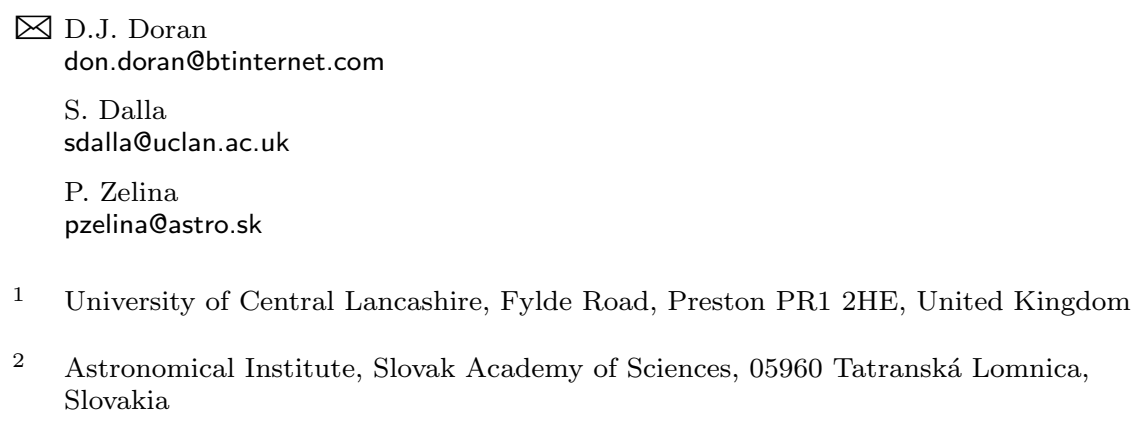


Keywords: Cosmic Rays, Solar; Energetic Particles, Abundances; Energetic Particles, Propagation

\section{Introduction}

Solar energetic particles (SEPs) are ions and electrons that have been accelerated from the coronal and solar wind plasma by solar flares and coronal mass ejections (CMEs), and that are detected in the space environment by instruments onboard spacecraft. SEP ions consist mainly of protons, with further small numbers of heavier ions from helium up to iron, and lesser amounts of the ion species heavier than iron. Solar flares and CME driven shocks typically accelerate SEPs to high kinetic energies which are sometimes relativistic at several $\mathrm{GeV}$ nucleon ${ }^{-1}$ for ions, and to tens of $\mathrm{MeV}$ for electrons. Normally, the energies are in the $\mathrm{keV}$ to $\mathrm{MeV}$ range. SEPs are of particular importance for scientific and spaceexploration missions, because they are a radiation hazard to spacecraft and to high-altitude human occupants. Of these radiation-hazard particles, protons are the most abundant, and they therefore present the most serious safety risk. In this regard, human occupants can suffer cumulative radiation dosages causing serious health issues, and spacecraft can experience irreversible damage to their functional electronics, (Reames, 1999; Kallenrode, 2004; Crosby et al., 2015).

Solar flares and CMEs have long been assumed to be separate solar events, as they can occur independently. Flares are not always associated with CMEs, and CMEs may not always have an associated flare, except in large SEP events when they usually occur together. Sometimes the flare precedes the CME, and other times it follows the CME, usually within an hour of each other. Recently many studies of flare and CME properties have been made by Aarnio et al. (2011); Shanmugaraju, Moon, and Vršnak (2011); Vršnak (2016); Compagnino, Romano, and Zuccarello (2017), using for CME data the Large Angle Spectrometric COronagraph (LASCO) onboard the Solar and Heliospheric Observatory (SOHO), and CME catalogs: one provided by the Coordinated Data Analysis Workshops (CDAW) Data Center and one obtained by the Computer Aided CME Tracking software (CACTus) detection algorithm during Solar Cycles 23 and 24. For flares they used a dataset containing 19,811 flares of C-, M-, and X-class observed by the Geostationary Operational Environmental Satellite (GOES) during the same period. These observations were also accompanied by Solar TErrestrial Relations Observatory (STEREO) data. Since both flares and CMEs accelerate SEPs, then SEPs can be used to study them. Historically, SEP events are separated into two classes according to whether the event is impulsive (flares) or gradual (CMEs) in a two-class paradigm developed by Reames (1995) and later revised by Cliver (2009). Typically, SEP events are studied by analyzing the profiles of particle intensity versus time, in what is commonly known as the time-intensity profile. One notable project for the analysis of SEP profiles is the Data Services and Analysis Tools for Solar Energetic Particle Events and Related Electromagnetic Emissions (SEPServer), which was a three-year collaborative project funded under the seventh framework programme (FP7-SPACE) of the European Union (Vainio et al., 2013). SEPServer constructed an internet server that provides 
access to a large number of SEP datasets from different instruments onboard half-a-dozen missions, including electromagnetic (EM) observations related to the SEP events. The project contains state-of-the art analysis tools that can be used to infer the solar eruption and SEP emission-time profiles.

The interplanetary transport of SEPs in the heliosphere from the source foot-point on the Sun to actual interaction with the Earth's magnetosphere, is determined largely by the interplanetary magnetic field (IMF). Whether or not a near-Earth observer will detect SEPs from a given SEP event depends on many factors. Multi-spacecraft observations at 1 AU have shown how SEP profiles and their properties depend on the location of the observing spacecraft with respect to the associated solar events (Cane, Reames, and von Rosenvinge, 1988; Kallenrode et al., 1993; Reames, Barbier, and Ng, 1996; Richardson et al., 2014). It is now known that near-Earth spacecraft are magnetically connected to solar locations on the western limb along the Parker spiral; therefore events within W20 - W80 have the largest probability to produce SEP events at Earth, according to Lario, Roelof, and Decker (2014). However, not all SEP events have their foot-points well connected to the magnetic-field lines at the observer's location, as has been shown by Gómez-Herrero et al. (2015); Lario et al. (2014); Reames, Ng, and Tylka (2013); and Wiedenbeck et al. (2013), who report that SEP events can be observed by multiple spacecraft locations even when their longitudinal separation is greater than 136 degrees. The effects of longitudinal separation of observer positions with respect to the event at the Sun has been reported by Kallenrode et al. (1993), and Wibberenz and Cane (1993), using multiple spacecraft data from Helios 1, Helios 2, and Interplanetary Monitoring Platform 8 (IMP 8), as well as by Lario et al. (2013) and Richardson et al. (2014), from the STEREO era. Recent observations of SEP events coming from compact solar sources by widely separated spacecraft by as much as $180^{\circ}$ in longitude as reported by Wiedenbeck et al. (2013) and Park et al. (2013) seem to imply significant cross-field transport, or movement across the interplanetary magnetic field lines. Using a model that accounts for the SEP propagation along field lines that meander as a result of plasma turbulence, (Laitinen et al., 2016) report that SEP propagation can be understood as a result of the effects of magneticfield-line random walk in the interplanetary medium, contrary to the diffusive model of SEP propagation. Some or all of this type of cross-field transport may take place close to the Sun, where shocks can accelerate particles across a wide range of the magnetic-field lines. Spacecraft located near-Earth are magnetically connected to solar locations on the western limb. On the other hand, the role of latitudinal separation between observers and solar source regions in determining whether or not an SEP event will be observed is less clear (Dalla et al., 2003; Dalla and Agueda, 2010; Rodríguez-Gasén et al., 2014).

Heavy-ion abundances in the heliosphere, apart from hydrogen, consist mainly of helium, with lesser amounts of $\mathrm{C}, \mathrm{N}, \mathrm{O}, \mathrm{Ne}, \mathrm{Mg}, \mathrm{Si}$, and $\mathrm{Fe}$, and with even lesser amounts of the ions heavier than Fe. In this article, we examine the intensities and spectra of He compared to $\mathrm{C}$, and $\mathrm{O}$ compared to $\mathrm{Fe}$, relative to those of protons. Abundances of heavy ions have been studied by Reames (1998); Mason, Mazur, and Dwyer (2002); Reames and Ng (2004); Desai et al. (2006); and Reames (2014), using the Wind spacecraft, and observations from 
Cosmic Ray Isotope Spectrometer (ULEIS) onboard Advanced Composition Explorer (ACE). In most studies the authors proposed scattering of the particles caused by the IMF that depends on the energy and the mass-to-charge ratio $[A / Q]$, for different species at the same velocity. Common ratios used for the $A / Q$-analysis are those of $\mathrm{Fe} / \mathrm{C}$ and $\mathrm{Fe} / \mathrm{O}$, which are of particular interest for this article. Previously, spacecraft and instrumentation sensitivity were not available to do comprehensive studies for heavy ions, and so the temporal dependence of the ratios such as $\mathrm{Fe} / \mathrm{O}$ and the spectra of ion species was not researched.

The energy spectra of heavy ions, showing the particle intensity at a given time versus the average energy of each instrument channel, provide an alternative view of SEP events. The heavy-ion spectra have been analyzed for a variety of SEP events by Reames, Ng, and Tylka (2001); Cohen et al. (2005); Tylka and Lee (2006); and Desai et al. (2016); however, in most studies the fluence of the particles is used to create a single representative spectrum. Reames et al. (1997) considered energy spectra at three time intervals during an SEP event observed by multiple spacecraft, and they showed that so-called invariant spectra are observed in the decay phase of the event, where the different spectra of each observing spacecraft at different positions become very similar to each other. Such event integrated spectra tend to exhibit a power-law dependence, which for large shock-associated gradual events almost always yields a double power law, sometimes called a rollover or knee. According to Li et al. (2009) and Martirosyan (2005) the energy break or rollover feature depends on $(Q / A)^{\alpha}$, where $Q$ is the ionic charge and $A$ is the ion mass in atomic mass units, with the power-law index $\alpha$ ranging between $\approx 1$ and $\approx 2$. The $Q / A$-dependence of the break energies however, is not always easy to determine due to the fact that the charge state, is very often uncertain, as shown by Mewaldt et al. (2012), who presented eventintegrated proton spectra for 16 GLE events over an energy range from $0.3 \mathrm{MeV}$ to $\approx 700 \mathrm{MeV}$. A more comprehensive study of the ratios of the heavy ions in $\mathrm{SEP}$ events, in particular the $\mathrm{Fe} / \mathrm{O}$ ratio, has been presented by Zelina et al. (2017).

A simulated propagation of iron ions injected near the Sun through interplanetary space with a variety of charge states uniformly distributed in energy by means of a 3D model has been constructed by Dalla, Marsh, and Battarbee (2017). The authors show that ions of different charge propagate with very different efficiencies to a location that is not magnetically well connected to the source. The authors conclude that drift-associated propagation is a possible explanation for the observed distribution measured in interplanetary space and cannot be taken to represent the injection at the source.

In this article we analyze the temporal evolution of heavy-ion spectra of $\mathrm{He}, \mathrm{C}$, $\mathrm{O}$, and $\mathrm{Fe}$, using a large number of snapshots and instrumentation from multiplespacecraft SEP events. Our focus is on two large events from the STEREO era with strong flux at both the low- and high-energy ends of the SEP range, to ensure that many spectral points with good statistics are available. We discuss the observations within the framework of the current understanding of SEP acceleration and propagation. 


\section{Event of 27 January 2012}

\subsection{Event of 27 January 2012 Properties}

Two SEP events followed each other in close succession on 23 and 27 January, as observed by STEREO, GOES-13, and SOHO spacecraft, providing a view from three vantage points. They were reported by the CDAW, HELIO, and SolarMonitor catalogs as having originated from two CMEs and their associated flares. The first event occurred on 23 January 2012 at 04:00 at N28W21, and is attributed to a halo CME with a median velocity of $1092 \mathrm{~km} \mathrm{~s}^{-1}$. It is associated with an M8.7 class flare, at N28W21, at NOAA Active Region (NAR) 11402, starting at 03:38. The second event, which is the event of interest shown in Figure 2, occurred on 27 January 2012, and it is attributed to a halo CME that started at 19:12 at N27W71, with a median velocity of $1133 \mathrm{~km} \mathrm{~s}^{-1}$. This CME is associated with an X1.7 flare starting at 17:37, from N21W71, at NOAA active region NAR 11402. In the first event (23 January 2012), the flare starts $\approx 22$ minutes before the CME, whereas in the second event (27 January 2012) it starts $\approx 83$ minutes before the CME. We discuss the second event in detail because it has the highest particle intensities at the higher energies, and therefore it is more likely to show the properties of the heavier ions such as oxygen and iron. However, the background fluxes for this event are high due to the preceding event. The positions of the STEREO spacecraft are shown in Figure 1, where they are separated from each other by $137.7^{\circ}$ and from Earth by over $100^{\circ}$. These spacecraft locations, along with those near Earth, provide an excellent opportunity to view the properties of particles arriving at $\approx 1 \mathrm{AU}$.

Both of these events were reviewed by Correia et al. (2013), who discuss the impact of SEPs arriving at the Earth's ionosphere and detected by riometers $(30-38.2 \mathrm{MHz})$, ionosonde, and VLF systems based in Antarctica for (GeV protons), and Brazil for (10-12 GeV protons). The event of 23 January, was studied by Gopalswamy (2012) and Joshi et al. (2013) using both space-borne and ground-based instruments. They attributed the event to flares and two CMEs as being responsible for one of the largest SEP events of cycle 24. The two CMEs occurred in rapid succession and interacted close to the Sun, resulting in a double-dip geomagnetic storm of moderate intensity due to the angle of propagation of the ICME. They concluded that even though some CMEs associated with large SEP events are very energetic, they seem to be less efficient in accelerating particles.

The second event of 27 January, according to Frahm et al. (2013), was associated with an X-class flare with a high-energy particle stream flowing along the IMF arriving at Mars in $\approx 39$ minutes with the resulting CME arriving at Mars several days later. The 27 January event was also studied by Watanabe et al. (2013) where the authors report white-light emissions from an X1.7 class flare using the Solar Optical Telescope (SOT) onboard the Hinode spacecraft. These observations allowed the temperature distribution to be calculated from the white-light data, which indicated that high-energy particles penetrated down to near the photosphere, depositing heat into the ambient lower layers of the Sun's atmosphere. 


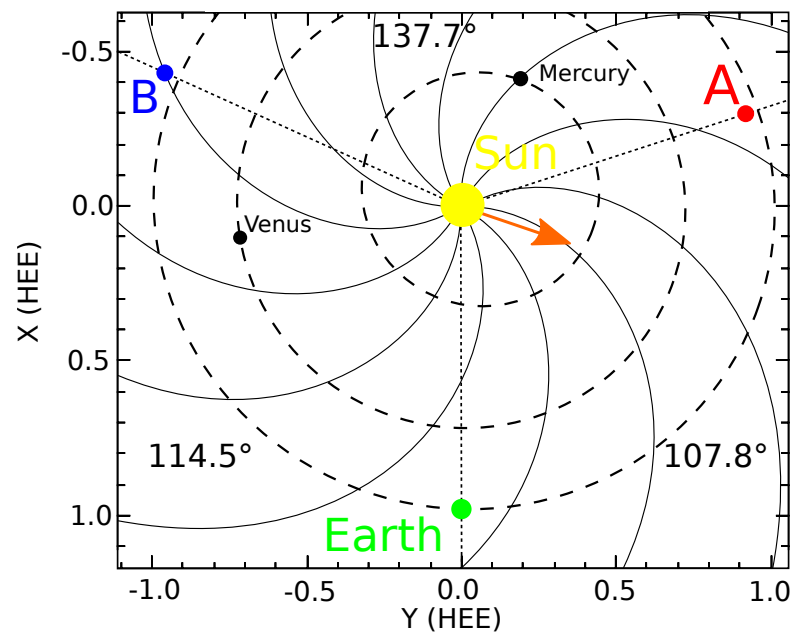

Figure 1. Orbital trajectories of STEREO-A and -B for the 27 January 2012 event, where the spacecraft locations are shown. The HEE notation is the Heliocentric Earth Ecliptic along the X- and Y- axis of the Earth-Sun line, and the Z- axis is pointed to the Ecliptic North Pole. The STEREO-A (ahead of the Earth) spacecraft is the red circle approximately on the Earth orbital path shown by the outermost dashed circle, while STEREO-B (behind the Earth) is the blue circle, and the Earth shown as the green circle. The angles of separation are: STEREO-B to Earth $=114.5^{\circ}$, STEREO-A to Earth $=107.8^{\circ}$, and STEREO-A to STEREO-B $=137.7^{\circ}$. This figure was adapted from the image at the NASA STEREO Science Center website at stereo-ssc.nascom.nasa.gov/where.shtm.

For the SEP event of 27 January 2012, we illustrate the fluxes for hydrogen, oxygen, and iron, and the evolution of the spectra for $\mathrm{He}, \mathrm{C}, \mathrm{O}$, and $\mathrm{Fe}$ as itemized below:

- Time-intensity profiles for hydrogen (Figure 2, measured at GOES-13) and for oxygen and iron (Figure 3, measured at ACE/SIS). The fluxes for oxygen (panel a) and iron (panel b) for the 27 January 2012 event are shown in Figure 3, where the numbered segments refer to the numbered panels of the montages of the spectral evolution of ion species in Figures 4 to 9 .

- Spectral plots for oxygen and iron from measurements at one location at all energies and instruments, as observed at near the Earth's position in Figures 4 and 5, plus measurements at STEREO-A and -B in Figures 6 and 7. The numerical reference to the temporal interval refers to the segments in Figure 3, where the numbers 1 through 7 provide an approximation of where that spectral snapshot would be represented on the time-intensity profile.

- Spectral plots containing the evolution of the spectra at three locations for helium and carbon (Figure 8), and for iron and oxygen (Figure 9). The numerical reference to the temporal interval refers to the segments in Figure 3 . 


\subsection{Heavy Ion Intensities Associated With the 27 January 2012 Event}

The proton intensities in particles $\mathrm{cm}^{-2} \mathrm{~s}^{-1} \mathrm{sr}^{-1}\left(\mathrm{MeV} \mathrm{nuc}^{-1}\right)^{-1}$ as shown in Figure 2, are orders of magnitude higher $\left(3 \times 10^{1}\right.$ in the $8-15 \mathrm{MeV}$ channel and $10^{0}$ in the $84-200 \mathrm{MeV}$ channel $)$ than those oxygen $\left(2 \times 10^{-2}\right.$ in the $10-13$ $\mathrm{MeV}$ channel and $10^{-4}$ in the $37-64 \mathrm{MeV}$ channel) and iron $\left(10^{-3}\right.$ in the $11-$ $16 \mathrm{MeV}$ channel and $5 \times 10^{-5}$ in the $36-52 \mathrm{MeV}$ channel) shown in Figure 3 . In Figure 2, the event of interest is the flux enhancement of 27 January 2012 (peak b), where the numbered segments correspond to those of Figures 3, and the numbered panels of Figures 4 to 9. In these figures, the time of the peak fluxes for the higher-energy particles occurs before those of the lower-energy particles, and there are far more particles at the lower energies. In addition, the peak fluxes for protons occurs before (28 January at 01:12) the peak flux for oxygen (28 January, 06:00) and for iron (28 January at 07:30). There is some considerable distribution of the particles in terms of energies, species, and arrival times at near-Earth spacecraft.

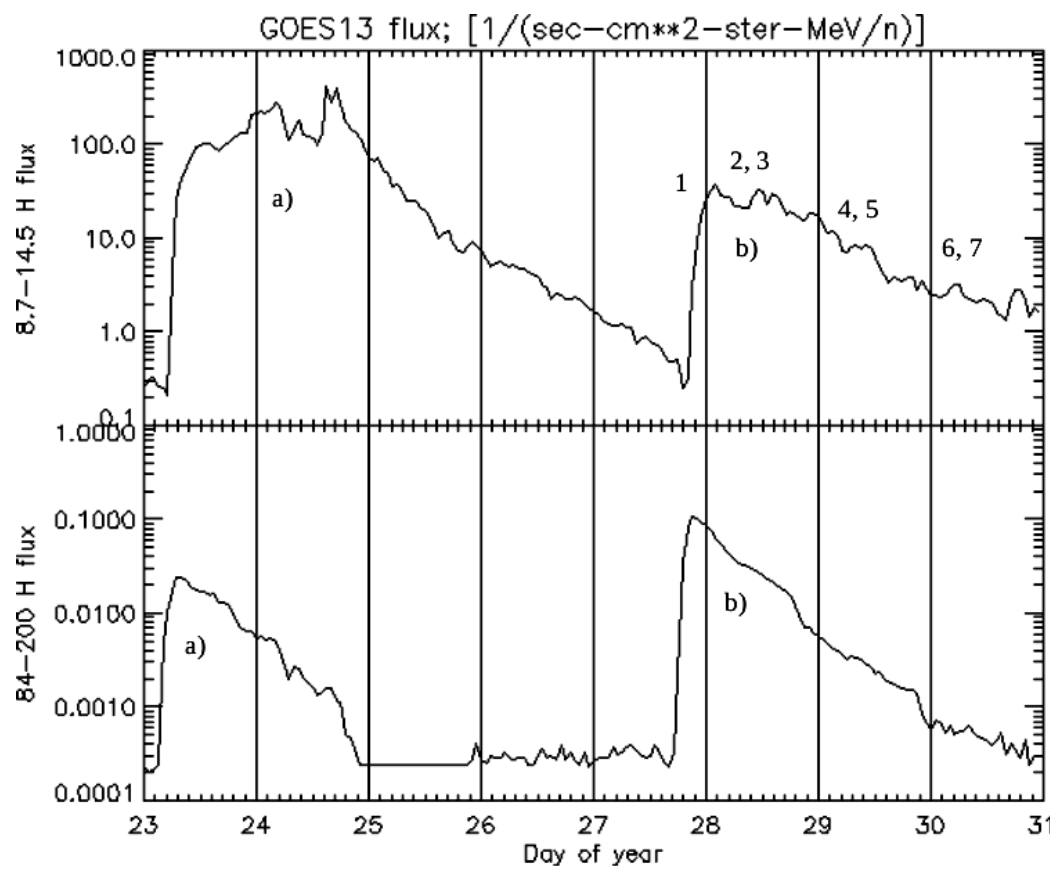

Figure 2. Proton fluxes for GOES-13 between 23 and 31 January 2012 showing the closely preceding event of 23 January (peak a) to the event of 27 January (peak $b$ ), which is the event of interest having higher fluxes at the higher energies. The numbered segments correspond to the numbered panels of the montages of the spectral evolution of species in Figures 3 to 9. 


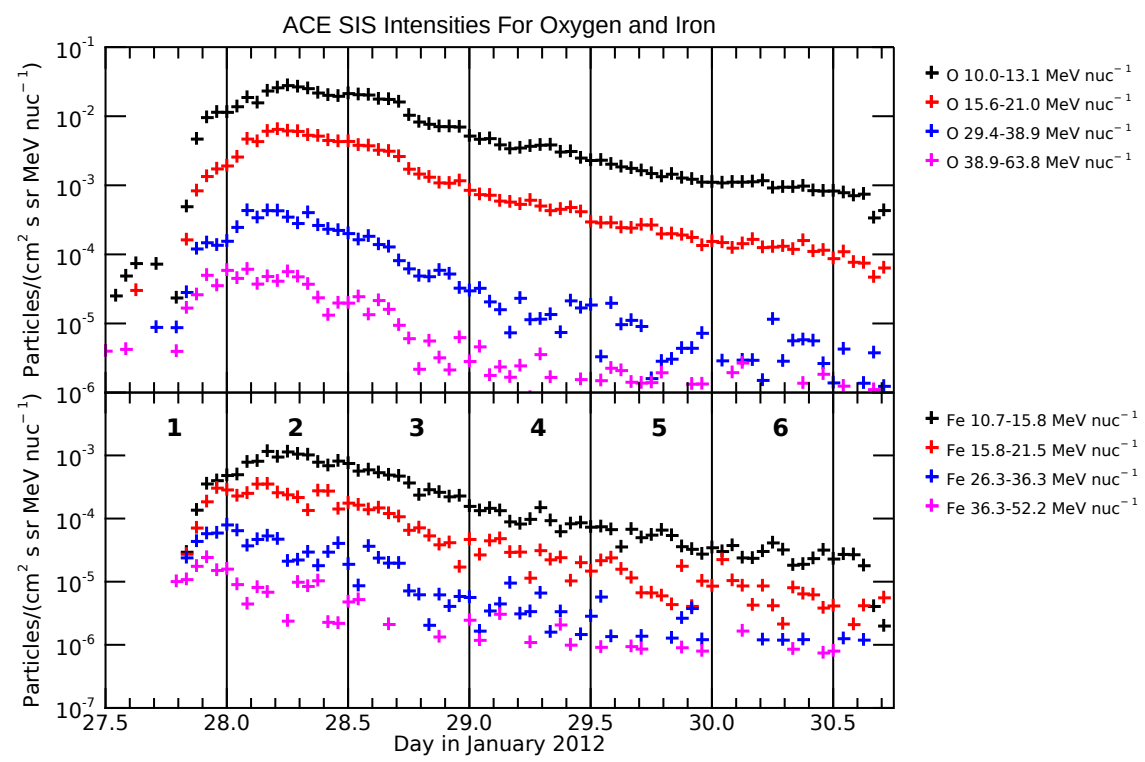

Figure 3. 27 January 2012 SEP event time-intensity profiles of oxygen (top) and iron (bottom) at ACE/SIS. The numbered segments 1 to 6 refer to 12-hour intervals, over which the temporal evolution of the spectral plots in Figures 4 to 9 were evaluated.

\subsection{Heavy Ion Spectra Associated With the 27 January 2012 Event}

Compared to the intensities of protons, the heavier ion intensities of He, C, $\mathrm{O}$, and $\mathrm{Fe}$ are orders of magnitude lower in the three heliospheric locations. For these observations, we have focused on near-Earth, STEREO-A, and STEREO-B locations to illustrate the differences in the spectral evolution of heavy ions.

\subsubsection{Oxygen Spectral Evolution at Near-Earth Locations}

Beginning with the spectra in Figure 4, for oxygen at near-Earth locations measured by Solar Isotope Spectrometer (SIS) and Ultra Low Energy Isotope Spectrometer (ULEIS) onboard ACE, the background flux rates are shown in panel a for ULEIS and SIS, but Electron, Proton, and Alpha Monitor (EPAM) and Cosmic Ray Isotope Spectrometer (CRIS) instruments onboard the same spacecraft are not recording particles for this set of nine panels. In the next panel $\mathrm{b}, \approx$ three hours later, the first particles arrive at the higher energies in the 20 to $100 \mathrm{MeV}$ channels. The particles at the higher energies continue to arrive in panel c, one hour later, but the lower-energy particles are yet to arrive. The particles at the lower energies $<10 \mathrm{MeV}$ do arrive in panel $\mathrm{d}$, $\approx$ four hours after the higher energy particles, where an "arch" structure has formed at the lower energies, and the higher energies are becoming a power-law shape. The arch structure disappears in panel $\mathrm{e}, \approx$ five hours later, with continued arrival 
of the lower energy particles, and the overall shape of the spectra are becoming a power law at both of the ACE instruments, ULEIS and SIS. By the interval at panel $\mathrm{g}$, the time when the peak intensities of particles have been reached, the spectra are becoming a power law late in the event at panels $\mathrm{h}$ and i. Overall, an arch structure moves through the spectra from the higher energies to the lower energies. This is very similar to the pattern observed for protons in previous work by Doran and Dalla (2016).

\subsubsection{Iron Spectral Evolution at Near-Earth Location}

This set of spectra shown in nine panels in Figure 5 develops in a similar manner to the oxygen spectra above, except that contributions from the Wind instrument are also plotted, but the EPAM and CRIS instruments are not recording particles for this set of nine panels. Again, an arch structure develops in panels d, e, and f, which later becomes a power law in panels $\mathrm{g}, \mathrm{h}$, and $\mathrm{i}$. The difference between the oxygen and the iron spectral evolution, is that the higher-energy iron particles, being heavier, arrive later by $\approx$ one hour in panel c compared to those of oxygen in panel $\mathrm{b}$ of Figure 4.

\subsubsection{Oxygen Spectral Evolution at STEREO-A and -B}

In Figure 6 the oxygen spectral evolution is illustrated in nine panels for both of the STEREO-A and STEREO-B locations for instruments Low-Energy Telescope (LET) and Suprathermal Ion Telescope (SIT), beginning with the background rate of fluxes in panel a. Here the fluxes for STEREO-B (in light blue) are an order of magnitude lower than STEREO-A, indicating that STEREO-B is less well connected to the magnetic-field lines. Panels b, c and d show that particles begin to arrive at the higher energies first at STEREO-A, but not at STEREOB. It is clear that the STEREO-spacecraft locations are less well connected than ACE at near-Earth, as the arch structure is less apparent in the spectra. Similarly as in the ACE spectra, the higher-energy particles at STEREO-A arrive first in panels c and d. STEREO-B seems not to observe any particles throughout this event.

\subsubsection{Iron Spectral Evolution at STEREO-A and $-B$}

Figure 7 illustrates the evolution of the iron spectra for STEREO-A and -B locations for instruments LET and SIT. Similar to the spectra for oxygen, STEREO-B seems not to record any iron nuclei for this event compared to STEREO-A. Panel a represents the background flux rates recorded only by STEREO-A, with STEREO-B not being well connected. The arch structure is not present in the spectra for iron, as there seem to be very few particles at these energies $(<60 \mathrm{MeV})$. Particles observed by STEREO-B in panel h late in the event are an order of magnitude lower in flux than for STEREO-A. 


\subsubsection{Spectral Plots Containing the Evolution of the Spectra at Three Locations for Helium and Carbon}

In Figure 8, we illustrate spectra as observed by three spacecraft, near Earth at ACE/SIS, STEREO-A/LET, and STEREO-B/LET for helium and carbon. All of the spectra in this panel are quite widely separated owing mainly to the differing species of ions and the wide separation of the spacecraft, with nearEarth and STEREO-A being better connected. Panel $b$ is the beginning of the flux enhancement at ACE/SIS for helium, but all other spectra remain the same. One hour later in panel c, there is a large increase in flux at all energies for $\mathrm{ACE} / \mathrm{SIS}$ helium and carbon, with all other spectra remaining the same. There is a continuing small increase in the fluxes at ACE and STEREO-A instruments throughout panels d, e, and f, finally peaking out in panel g. An arch structure does begin to develop early for ACE/SIS in panel $b$ at the beginning of the flux enhancement, where it straightens in panel c, and again arches in panel d. The spectra remain widely separated into two groups for both species and spacecraft location, and taking on a power-law shape late in the event at panels g, h, and i.

\subsubsection{Spectral Plots Containing the Evolution of the Spectra at Three Locations for Oxygen and Iron}

Panel a in Figure 9, represents the background fluxes, with all the spectra closely grouped in a power-law shape, unlike the spectra of helium and carbon, which were widely separated. Most prominent are the spectra at STEREO-A for oxygen (red squares, SIT) and (light blue circles, LET). There is little change in panel $\mathrm{b}$ three hours later at the beginning of the flux enhancement. The main flux occurs and panel $\mathrm{c}$ at ACE/SIS for oxygen (black crosses) and ACE/SIS for iron (pink crosses), with all other spectra remaining the same. In panel $d$ there is an increase in STEREO-A flux for oxygen and iron at the higher energies, with spectra becoming generally harder in panels e and $\mathrm{f}$. The peak fluxes are observed in panel g, apart from those of STEREO-B at the bottom of the plot, which are not connected to the event. Late in the event, in panels $h$ and $i$, the spectra take on a power-law shape and become more closely grouped, with an arch structure for oxygen and iron taking shape in panels e, $\mathrm{f}, \mathrm{g}$, and $\mathrm{h}$ at STEREO-A/LET instruments (light blue and green colors). 
1

2

3

4

5

6

7

8

9

10

11

12

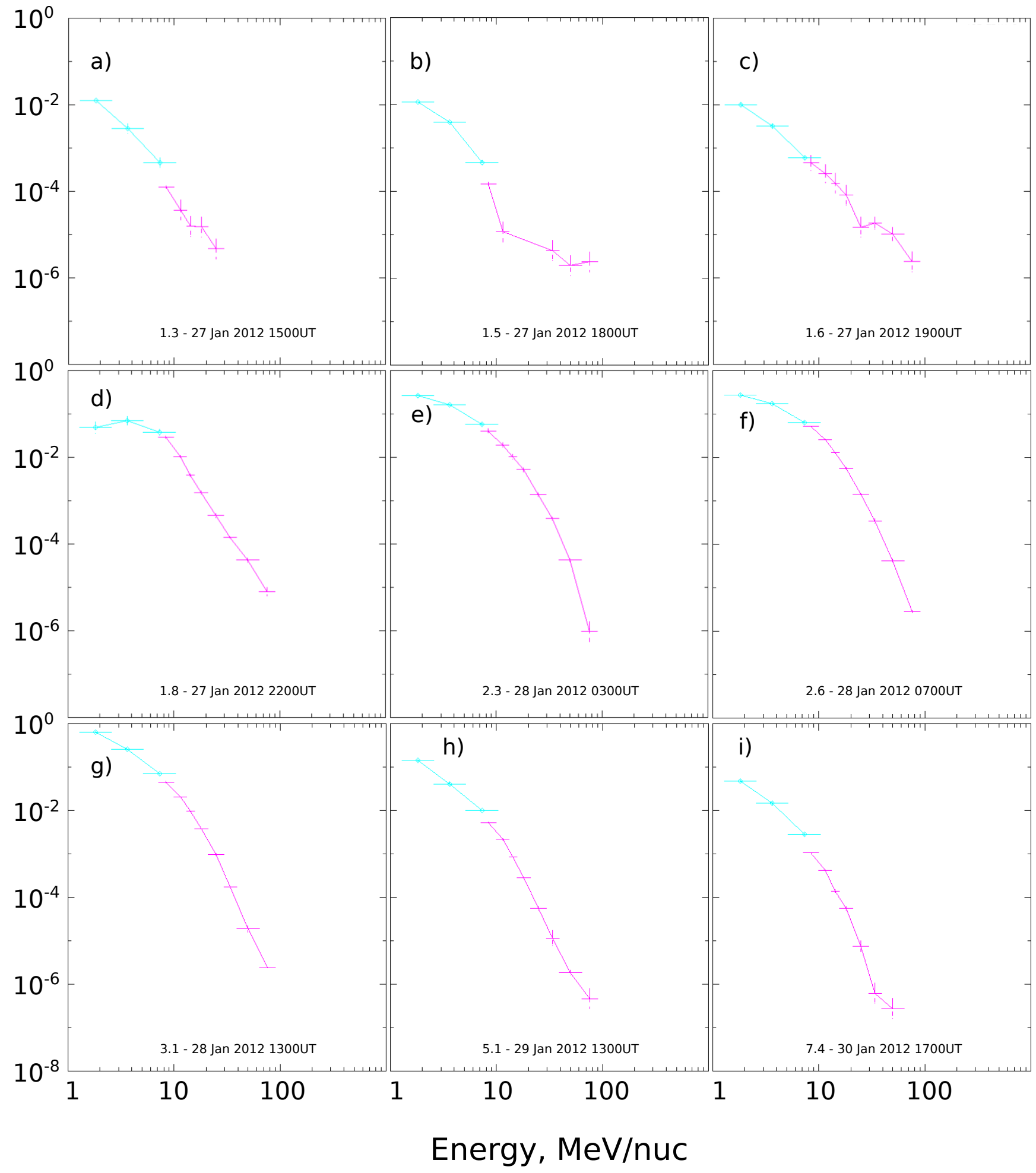

Figure 4. 27 January 2012 SEP event temporal evolution of oxygen energy spectra for Earth-located instruments ACE/SIS and ACE/ULEIS, where the numbered panels a) to i) refer to the 12-hour time intervals of the numbered segments of Figure 3. We show ACE/SIS in pink, and ACE/ULEIS in light blue. 
1

2

3

4

5

6

7

8

9

10

11

12

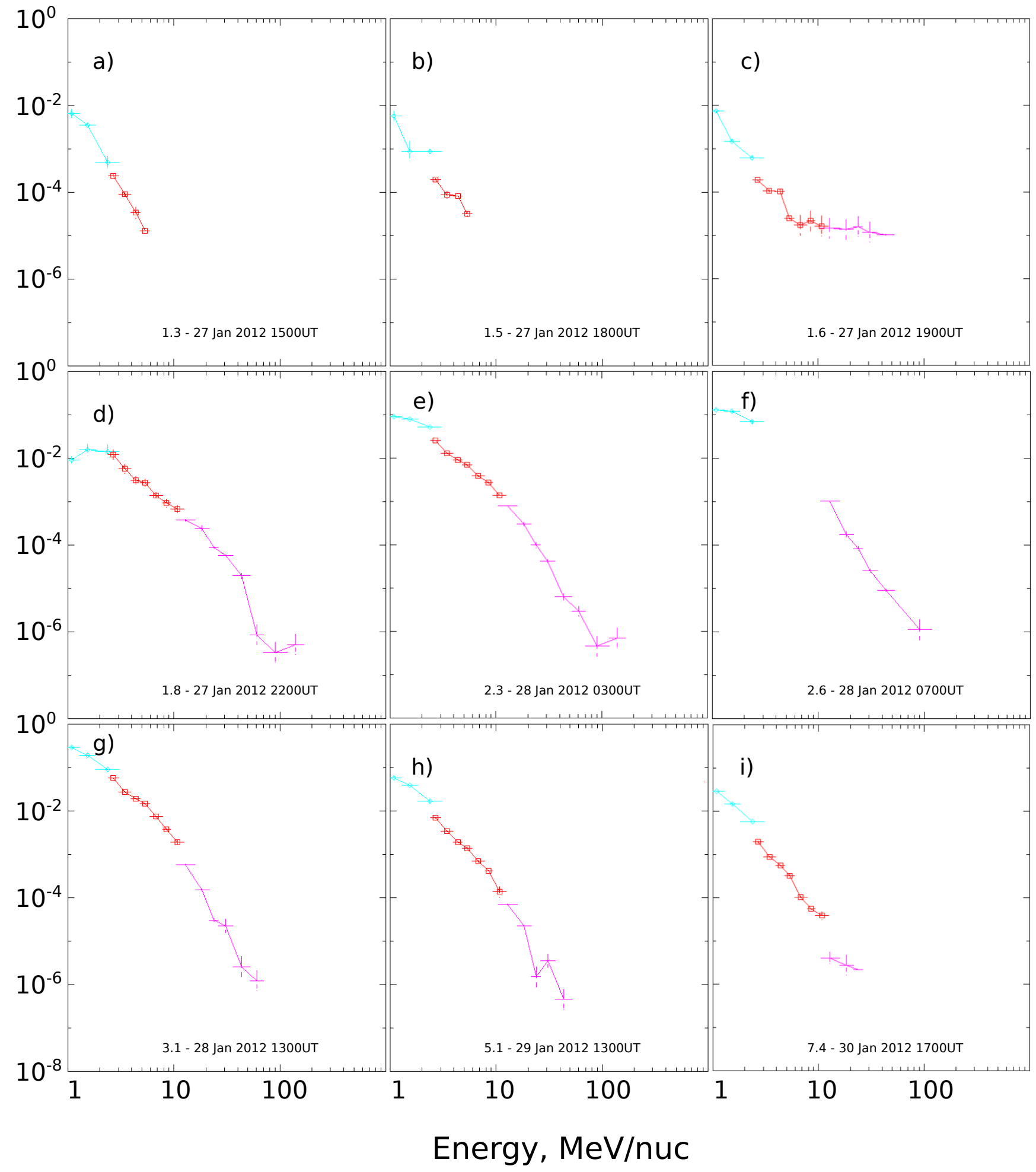

Figure 5. 27 January 2012 event temporal evolution of iron energy spectra for Earth-located instruments ACE/SIS and ACE/ULEIS, where the numbered panels a) to i) refer to the 12-hour time intervals of the numbered segments of Figure 3. ACE/SIS is plotted in pink, ACE/ULEIS in light blue, and Wind/EPACT plotted in red. 
Figure 6. 27 January 2012 event temporal evolution of oxygen energy spectra for STEREO-A and -B located instruments (STEREO-A/SIT and LET, STEREO-B/SIT and LET), where the numbered panels a) to $\mathrm{i}$ ) refer to the 12-hour time intervals of the numbered segments of Figure 3. STEREO-A/LET is plotted in black, STEREO-A/SIT in pink, STEREO-B/LET in light blue, and STEREO-B/SIT in green. 
1

2

3

4

5

6

7

8

9

10

11

12

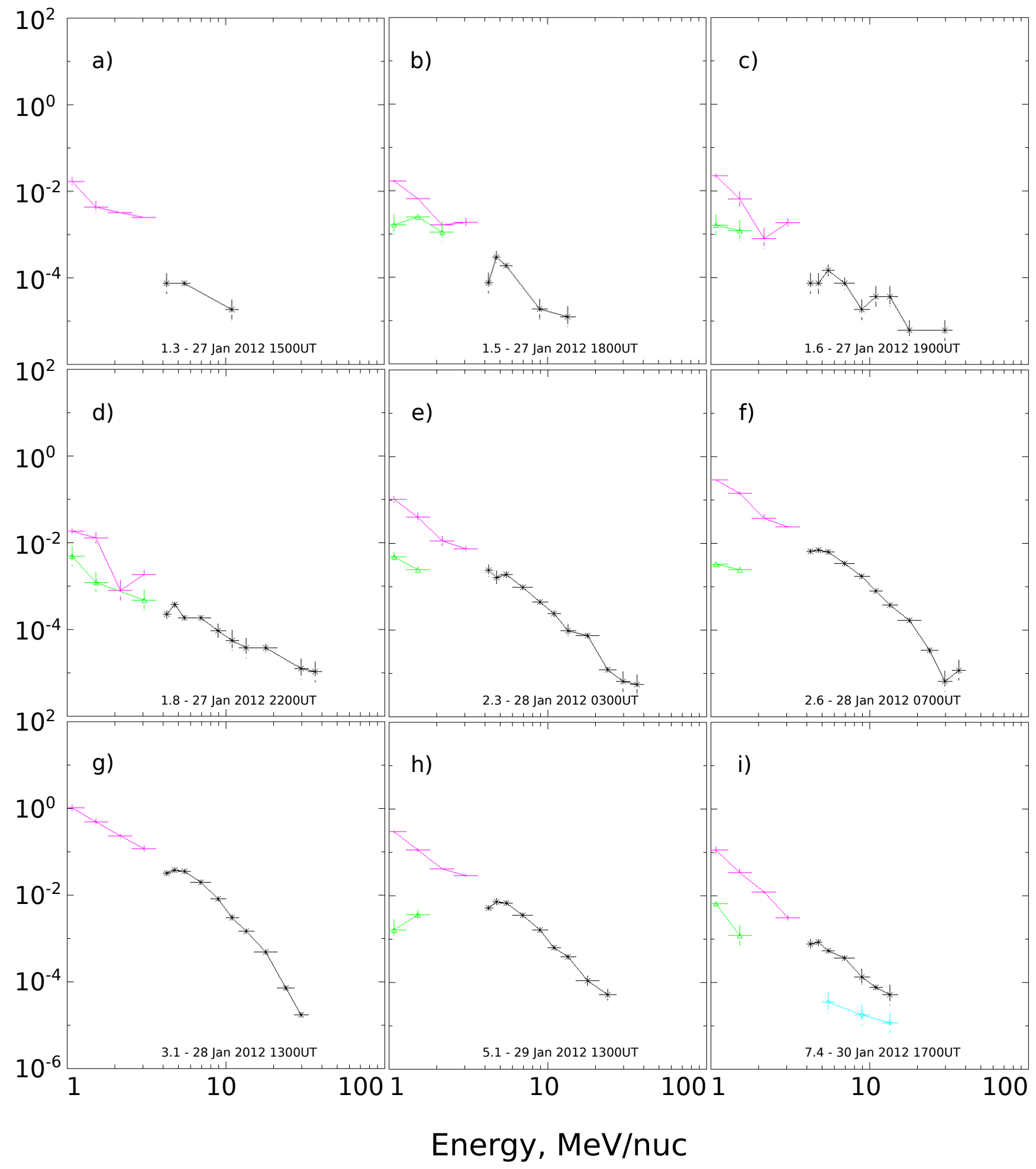

Figure 7. 27 January 2012 event temporal evolution of iron energy spectra for STEREO-A and -B located instruments (STEREO-A/SIT and LET, STEREO-B/SIT and LET), where the numbered panels a) to i) refer to the 12-hour time intervals of the numbered segments of Figure 3. STEREO-A/LET is plotted in black, STEREO-A/SIT in pink, STEREO-B/LET in light blue, and STEREO-B/SIT in green. 


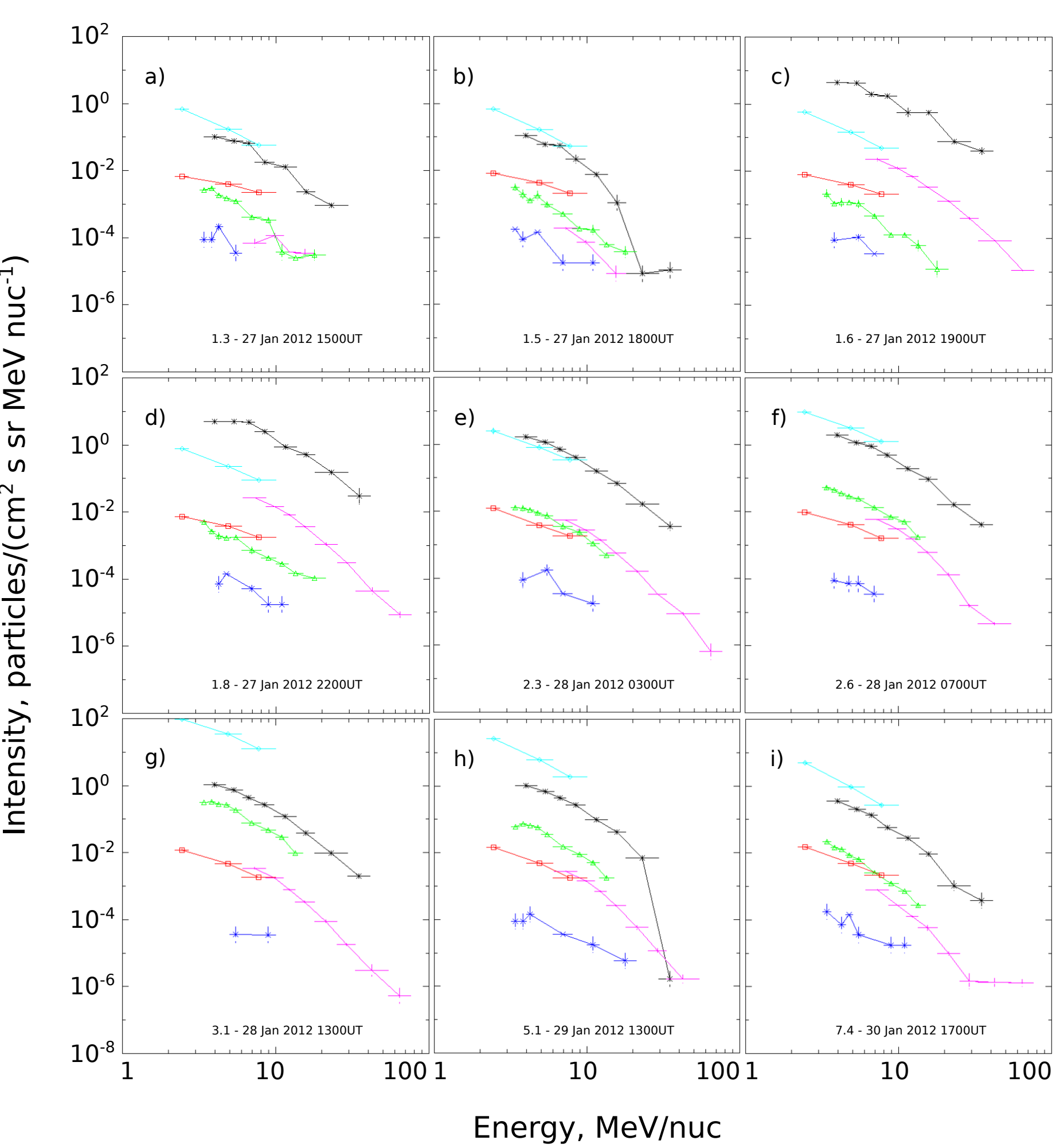

Figure 8. 27 January 2012 event temporal evolution of the He and C energy spectra for Earth-located instruments ACE/SIS, and STEREO-A and -B located instruments, where the numbered panels a) to i) refer to the 12-hour time intervals of the numbered segments of Figure 3. ACE/SIS for He is plotted in black, ACE/SIS for C in pink, STEREO-A/LET for He in light blue, STEREO-A/LET for C in green, STEREO-B/LET for He in red, and STEREO-B/LET for $\mathrm{C}$ in blue. 


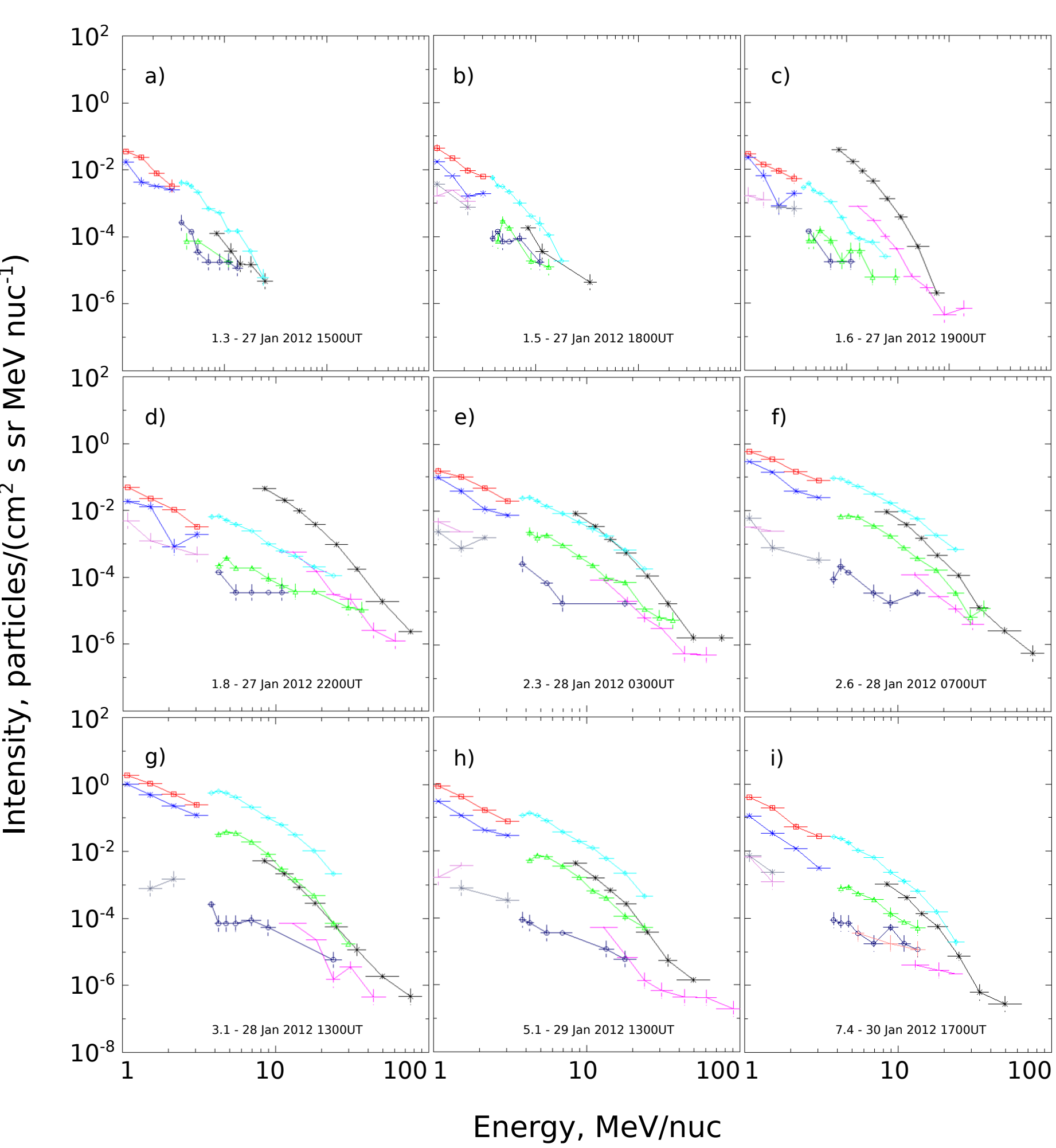

Figure 9. 27 January 2012 event temporal evolution of the $\mathrm{O}$ and $\mathrm{Fe}$ energy spectra for near-Earth located ACE/SIS, and STEREO-A and -B located instruments, where the numbered panels a) to i) refer to the 12-hour time intervals of the numbered segments of Figure 3. $\mathrm{ACE} / \mathrm{SIS}$ for $\mathrm{O}$ is plotted in black, ACE/SIS for $\mathrm{Fe}$ in pink, STEREO-A/LET for $\mathrm{O}$ in light blue, STEREO-A/LET for Fe in green, STEREO-A/SIT for O in red, STEREO-A/SIT for $\mathrm{Fe}$ in blue, STEREO-B/LET for $\mathrm{O}$ in dark blue, STEREO-B/LET for Fe in orange, STEREO-B/SIT for O in grey, and STEREO-B/SIT for Fe in magenta. 


\section{Event of 7 January 2014}

\subsection{Event of 7 January 2014 Properties}

The 7 January 2014 event occurred during the declining phase of Solar Cycle 24, when few X-class flares with SEPs were observed. High-energy particles of this double-peak event of 6 and 7 January, as shown in Figure 10 for proton fluxes, coincide with M- and X-class flares. However, Mays et al. (2015) reported that this flare and associated $2500 \mathrm{~km} \mathrm{~s}^{-1} \mathrm{CME}$ from a region close to the center of the Sun's disc, did not produce a geomagnetic storm as predicted in $\approx 36$ hours. The authors point out the importance of three-dimensional fitting of CMEs with three-dimensional imaging by multiple spacecraft. On the other hand, the event of 6 January, preceding the main event, did produce a GLE according to Thakur et al. (2014), one of only two GLEs in Cycle 24. The second event (peak b) in Figure 10 for 7 January 2014 has been expanded in Figure 11 for the proton fluxes at energy channels $8.7-14.5 \mathrm{MeV}, 16-40 \mathrm{MeV}, 36-82 \mathrm{MeV}$, and $81-200$ $\mathrm{MeV}$ from top to bottom. The numbered segments in each panel refer to the evolution of the spectra of the heavy ions shown in the spectral montages of Figures 14 to 19 .

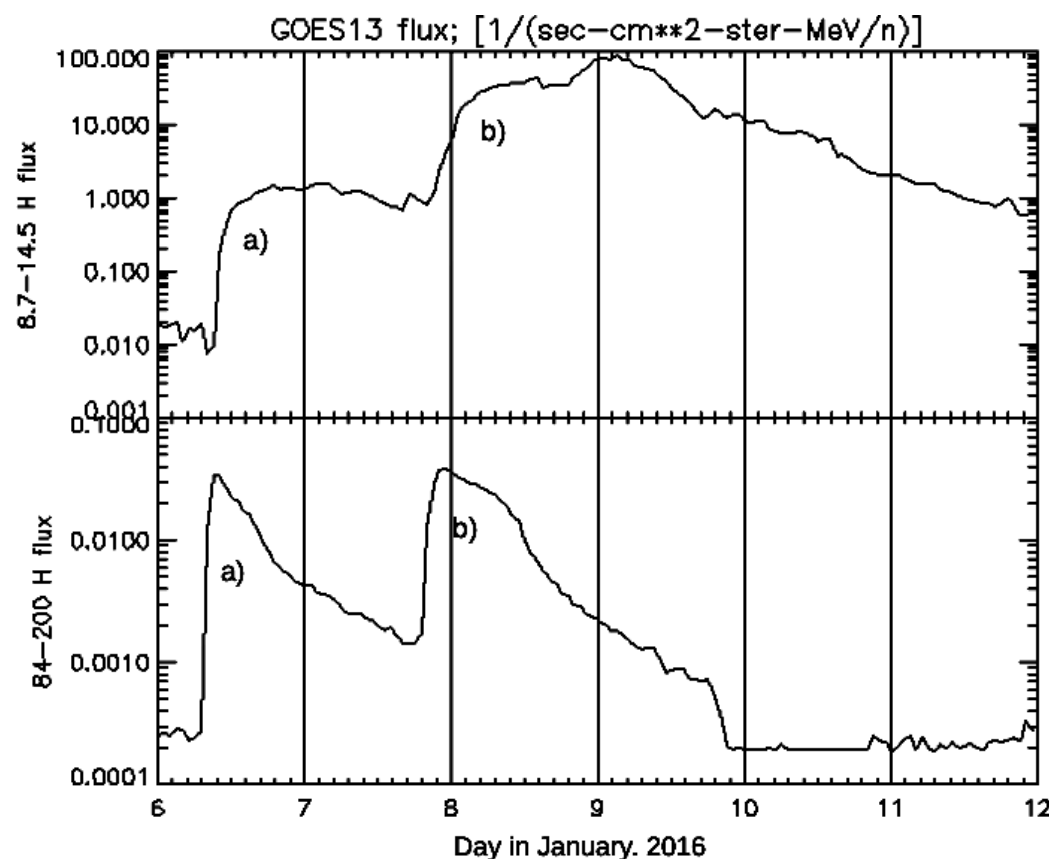

Figure 10. 6-12 January 2014 proton fluxes for GOES-13 showing two closely following events of 6 and 7 January. The event of interest is shown as peak $b$, which has a flux enhancement at $\approx 19: 00$ on 7 January, as detailed in Figure 11 for protons, and Figure 13 for iron and oxygen fluxes. 
Figure 11. 7-12 January 2014 proton fluxes for GOES-13, showing from top to bottom, energy channels 8.7-14.5 MeV, 16-40 MeV, 36-82 MeV, and 81-200 MeV. The numbered segments refer to the periods over which the fluxes are summed to obtain the temporal evolution of the spectra in Figures 14 to 19.

According to the CDAW, HELIO, and SolarMonitor catalogs this so-called "double event" originated from two CMEs and their associated flares. The first event occurred on 6 January 2014 at 09:12 from NAR 11944 at S15W11, and is attributed to a halo CME with a maximum velocity of $1953 \mathrm{~km} \mathrm{~s}^{-1}$. It is associated with a C- to M-class flare following the CME at 10:07 at S04E13 from the same active region. The second event, which is the event of interest shown in Figure 10 (peak b), occurred on 7 January 2014 at 18:36 from S14W11, and was attributed to a halo CME with a maximum velocity of $1926 \mathrm{~km} \mathrm{~s}^{-1}$. This CME was associated with an X1.2 class flare preceding the CME at 18:04, from S15W07, also at NAR 11944. In the first event (6 January 2014), the flare starts $\approx 55$ minutes after the CME, whereas in the second event (7 January 2014) it starts $\approx 32$ minutes before the CME. We discuss the second event, which has greater fluxes in the high energy channels, and therefore is more likely to show the properties of the heavy ions. However, the background fluxes for this event are high due to the preceding event. The positions of the STEREO spacecraft are shown in Figure 12.

As with the SEP event of 27 January 2012 in Section 3, we discuss the fluxes for hydrogen, oxygen, and iron, and the temporal evolution of the spectra for 


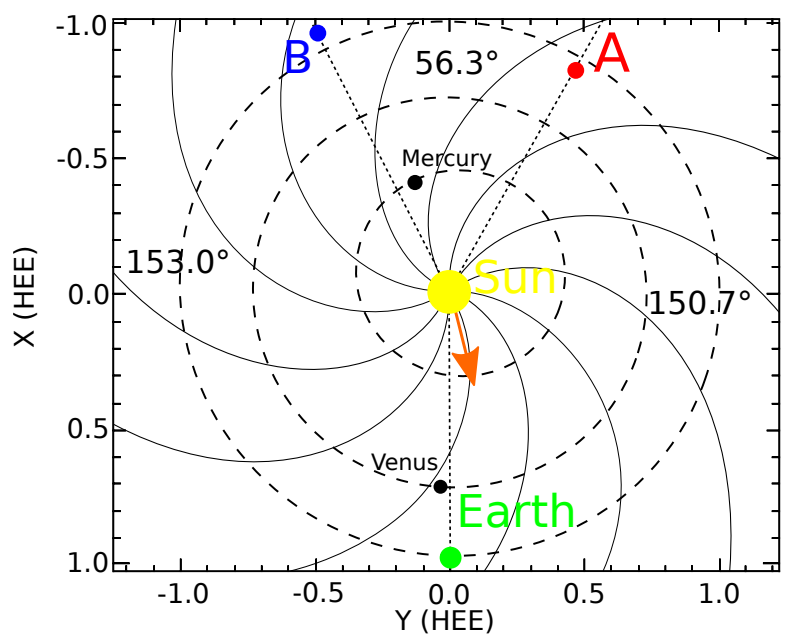

Figure 12. Orbital trajectories of STEREO-A and -B for the 7 January 2014 event, where the spacecraft locations are shown. The HEE denotes the Heliocentric Earth Ecliptic along the Xand Y-axis of the Earth-Sun line, and the Z-axis is the Ecliptic North Pole. The STEREO-A (ahead of the Earth) spacecraft is the red circle approximately on the Earth's orbital path of the dashed circle, while STEREO-B (behind the Earth) is the blue circle, and the Earth shown as the green circle. The angles of separation are: STEREO-B to Earth $=153.0^{\circ}$, STEREO-A to Earth $=150.7^{\circ}$, and STEREO-A to STEREO-B $=56.3^{\circ}$. This figure was adapted from the image at the NASA STEREO Science Center website at stereo-ssc.nascom.nasa.gov/where.shtm.

helium, carbon, oxygen, and iron for the SEP event of 7 January 2014 as itemized below:

- Time-intensity profiles for protons (Figures 10 and 11 measured at GOES13) and for oxygen and iron (Figure 13, measured at ACE/SIS). In Figure 13, the fluxes for oxygen (panel a) and iron (panel b) for the 7 January 2014 event show numbered segments that refer to the numbered panels of the montages of the spectral evolution of ion species in Figures 14 to 19.

- Spectral plots for the temporal evolution of oxygen and iron from measurements at one spacecraft location at $1 \mathrm{AU}$ at all energies and instruments, as observed at the near-Earth position at the ACE spacecraft in Figures 14 and 15, plus similar measurements at STEREO-A and -B LET and SIT instruments in Figures 16 and 17. The numerical reference to the temporal interval refers to the segments in Figure 13, where the numbers 1 through 6 provide an approximation of where that spectral snapshot would be represented on the time-intensity profile.

- Spectral plots containing the evolution of the spectra at the three locations of STEREO-A and -B, and ACE for helium and carbon (Figure 18), and for iron and oxygen (Figure 19). The numerical reference to the temporal interval refers to the segments in Figure 13. 
The proton intensities in particles $\mathrm{cm}^{-2} \mathrm{~s}^{-1} \mathrm{sr}^{-1}\left(\mathrm{MeV} \mathrm{nuc}^{-1}\right)^{-1}$ as shown in Figures 10 and 11 are orders of magnitude higher than for oxygen and iron as shown in Figure 13 for the same particle energies. In these figures, the peak fluxes for the higher energy particles occur before those of the lower energy particles, and there are far more particles at the lower energies. In addition, the peak flux for protons occurs later (9 January at 01:00) than the peak flux for oxygen (8 January at 03:00, 22 hours earlier), and for iron on (8 January at 00:00, 25 hours earlier), in similar energy channels for the near-Earth locations of GOES and ACE respectively. This peak time difference is due mainly to the abundance and energy of the ions compared to protons and their respective arrival times whereby the higher energy particles arrive earlier. There is a distribution of the particles in terms of energies, species, and arrival times at near-Earth spacecraft.

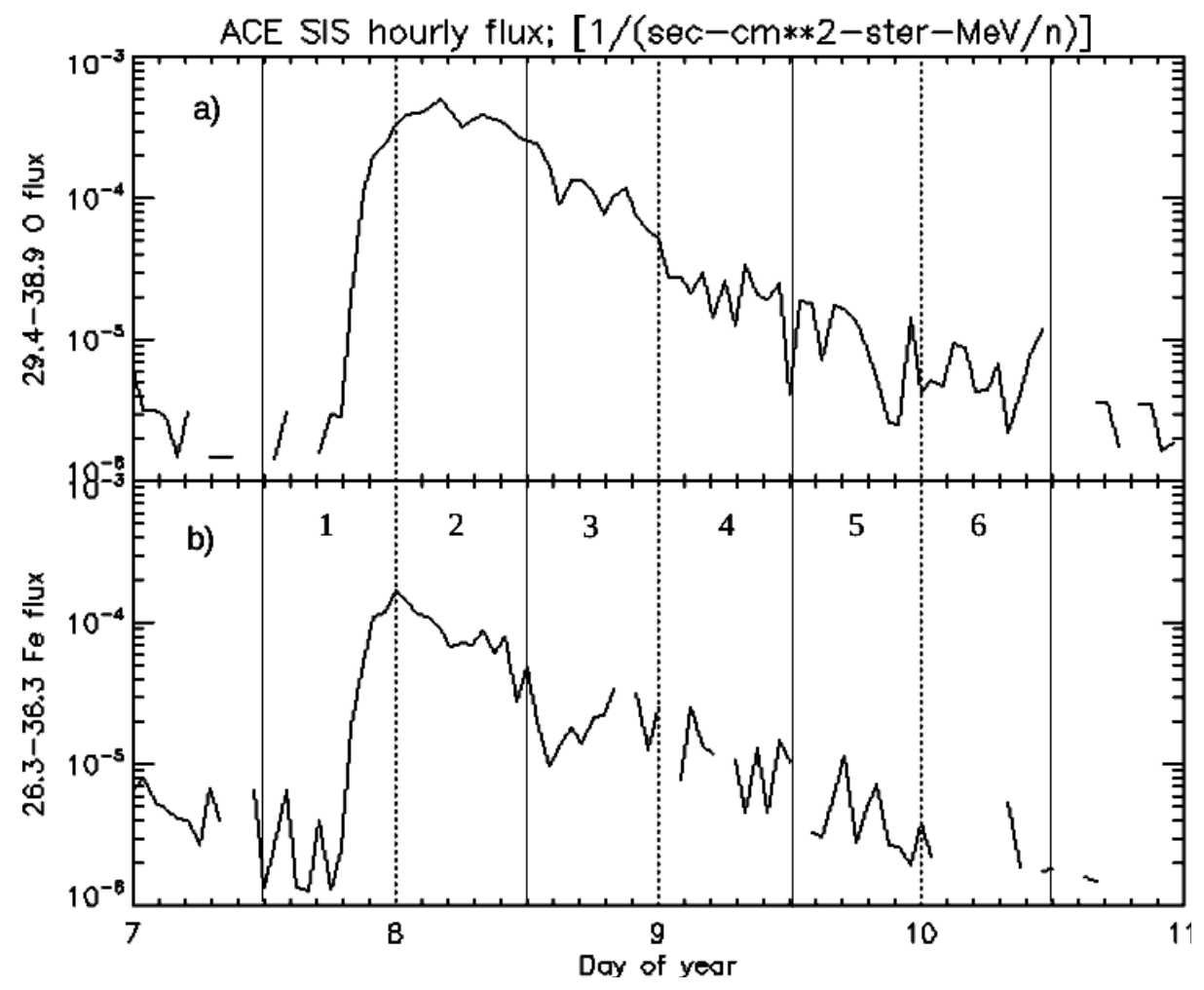

Figure 13. 7 January 2014 SEP event time-intensity profiles of oxygen and iron for ACE/SIS in particles $\mathrm{cm}^{-2} \mathrm{~s}^{-1} \mathrm{sr}^{-1}\left(\mathrm{MeV} n u c^{-1}\right)^{-1}$ in the top panel a for oxygen at energy channels $29.4-38.9 \mathrm{MeV}$, and bottom panel $b$ for iron at 28.3-36.3 MeV. The numbered segments 1 to 6 refer to the temporal evolution of the spectral plots in Figures 14 to 19. 


\subsection{Heavy Ion Spectra Associated with the 7 January 2014 Event}

Similar to the SEP event of 27 January 2012 discussed above, heavy ions of $\mathrm{He}, \mathrm{C}, \mathrm{O}$, and $\mathrm{Fe}$ of the event of 7 January 2014 are orders of magnitude lower in intensities in three heliospheric locations than those of protons. For these observations, we have focused on near-Earth, STEREO-A, and STEREO$\mathrm{B}$ locations to illustrate the differences in the spectral evolution of the heavy-ion spectra as detailed in the sections below.

\subsubsection{Oxygen Spectral Evolution at Near-Earth Locations}

The temporal evolution of the oxygen spectra is illustrated in the spectral montage in Figure 14, where a clear wave pattern develops from the highest energies to the lowest, creating an arch structure in panels d, e, and f. This is very similar to the pattern observed for protons in previous work by Doran and Dalla (2016). In this figure, we use ACE/SIS, ACE/ULEIS, and Wind/EPACT near-Earth locations for a well-connected energetic SEP event.

\subsubsection{Iron Spectral Evolution at Near-Earth Locations}

The iron spectral evolution is shown in Figure 15, where the spectra again reveal an arch shape developing from the highest energies to the lowest in panels d, e, and $\mathrm{f}$, but this is somewhat less obvious than the arch pattern for oxygen. This could be due to the lower intensities for iron ions and the fact that the particles arrive $\approx$ one hour later than the oxygen ions. In this figure, we use ACE/SIS, ACE/ULEIS, and Wind/EPACT at near-Earth locations for a well-connected, energetic SEP event.

\subsubsection{Helium and Carbon Ions Spectral Evolution at the Three Widely Separated Locations of ACE and STEREO-A and $-B$}

In Figure 18 we illustrate the spectra as observed by three spacecraft, near Earth at ACE/SIS, and widely separated spacecraft STEREO-A/LET, and STEREO-B/LET. In the first panel a the background fluxes are represented for all spacecraft, with the helium ion spectra being grouped at the top two spectra of ACE/SIS and STEREO-A/LET, separated by at least an order of magnitude lower flux for carbon ions of ACE/SIS and STEREO-A/LET. The spectra of STEREO-B/LET for helium ions are also in the lower group, but due to the spacecraft position it is poorly connected to this event, and it registers lower flux for helium ions than STEREO-A by at least one order of magnitude. In panels $\mathrm{c}$ and $\mathrm{d}$, the two groups are separated, but the higher energy particles begin to arrive in the $10-100 \mathrm{MeV}$ energy channel. In panels $\mathrm{d}$, e, and $\mathrm{f}$, the two groups remain separated, with flux increases at the higher energies at all locations. An arch shape begins to develop at ACE/SIS for helium ions (black) and ACE/SIS for carbon ions (pink) in panels d, e, and f. The arch structure is less obvious for the STEREO-A observations, but it does seem to track the general motions of the arch evolution for ACE/SIS, although it is very limited in the higher-energy channels. STEREO-B is not connected, and therefore fewer particle intensity increases are observed. 


\subsubsection{Oxygen and Iron Spectral Evolution at Three Widely Separated Locations} of $A C E$ and $S T E R E O-A$ and $-B$

In this illustration of the temporal evolution of the oxygen and iron comparison as shown in Figure 19, the background spectra are all very similar in panel a, but they are grouped orders of magnitude lower in intensities than those of helium and carbon above in Figure 18. Particles begin to arrive at the higher energies in panels $\mathrm{c}$ and d, with ACE/SIS for both oxygen and iron which are becoming separated from those of STEREO-A, and with STEREO-B, being not well connected to the event, making no particle-intensity observations. A slight arch structure develops in panels d, e, and $\mathrm{f}$ at ACE/SIS for oxygen ions, but less so for the iron ions. 


\section{Discussion and Conclusions}

We have selected two large SEP events from the STEREO era to illustrate the evolution of the heavy-ion spectra associated with the events. The two events, 27 January 2012 and 7 January 2014, were selected based on their high intensities for oxygen and iron at high energies, thus allowing a better chance to observe their spectral properties. We observed some features that the heavy-ion spectral evolution curves had in common with those of protons from earlier studies (Doran and Dalla, 2016). Some common features that we observed are as follows:

- When measuring the spectra of He, C, O, and Fe at three widely spread locations, i.e., STEREO-A and -B, and near Earth in this study, the spectra are widely separated according to groups based on species, and the observer's location. In this regard, ACE/SIS for helium was grouped with STEREO-A for helium, whereas the heavier carbon ions were in the lowerintensity grouping for the event of 27 January 2012. As for the oxygen and iron ions in the same event, the groupings were less separated with oxygen being the upper group at higher intensities, and iron being the lower. In this event, STEREO-B was not well connected the eruption of the flare. A very similar pattern for the spectral evolution was also observed for the event of 7 January 2014, where STEREO-B was also not well connected. In these spectra, a wave-like pattern moves from the higher to the lower energies, with an "arch" structure developing $\approx 10$ hours after the start of the event, which then straightens into a power law shape late in the event after $\approx 24$ hours, with widely separated groups according to species.

- The spectra measured at near-Earth alone, and STEREO-A and -B alone, gave a similar wave pattern together with an arch structure, although this was less pronounced than the spectral evolution of protons. In these spectra, the decay phase late in the event is characterized by a power law after $\approx$ 24 hours from the start of the event.

A number of processes influence the spectral evolution of the heavy ions as they propagate from the source, through the IMF to the observers location:

- The acceleration process for particle release at the source is energy and time dependent, affecting individual ions differently.

- The scattering of the particles during propagation through the IMF depends on the energy and the mass-to-charge ratio $[A / Q]$, for different species at the same velocity. According to Parker (1965), ions will diffuse out along the IMF with mean free paths dependent on rigidity, which could explain the late arrival of low-energy particles.

- The deceleration of particles during their propagation as a result of adiabatic deceleration, e.g., Ruffolo (1995), and drift-induced deceleration, e.g., Kóta (1979) and Dalla, Marsh, and Laitinen (2015).

Acknowledgements We thank the instrument teams for providing the data used in this study. We acknowledge use of the VEPO analysis tool as well as data and services provided by SEPEM and SEPSERVER. S. Dalla acknowledges support from STFC grant ST/M00760X/1. P. Zelina acknowledges support from the project VEGA 2/0004/16. 
D.J. Doran et al.

\section{Disclosure of Potential Conflict of Interests}

The authors declare that they have no conflicts of interest. 
1

2

3

4

5

6

7

8

9

10

11

12

13

14

15

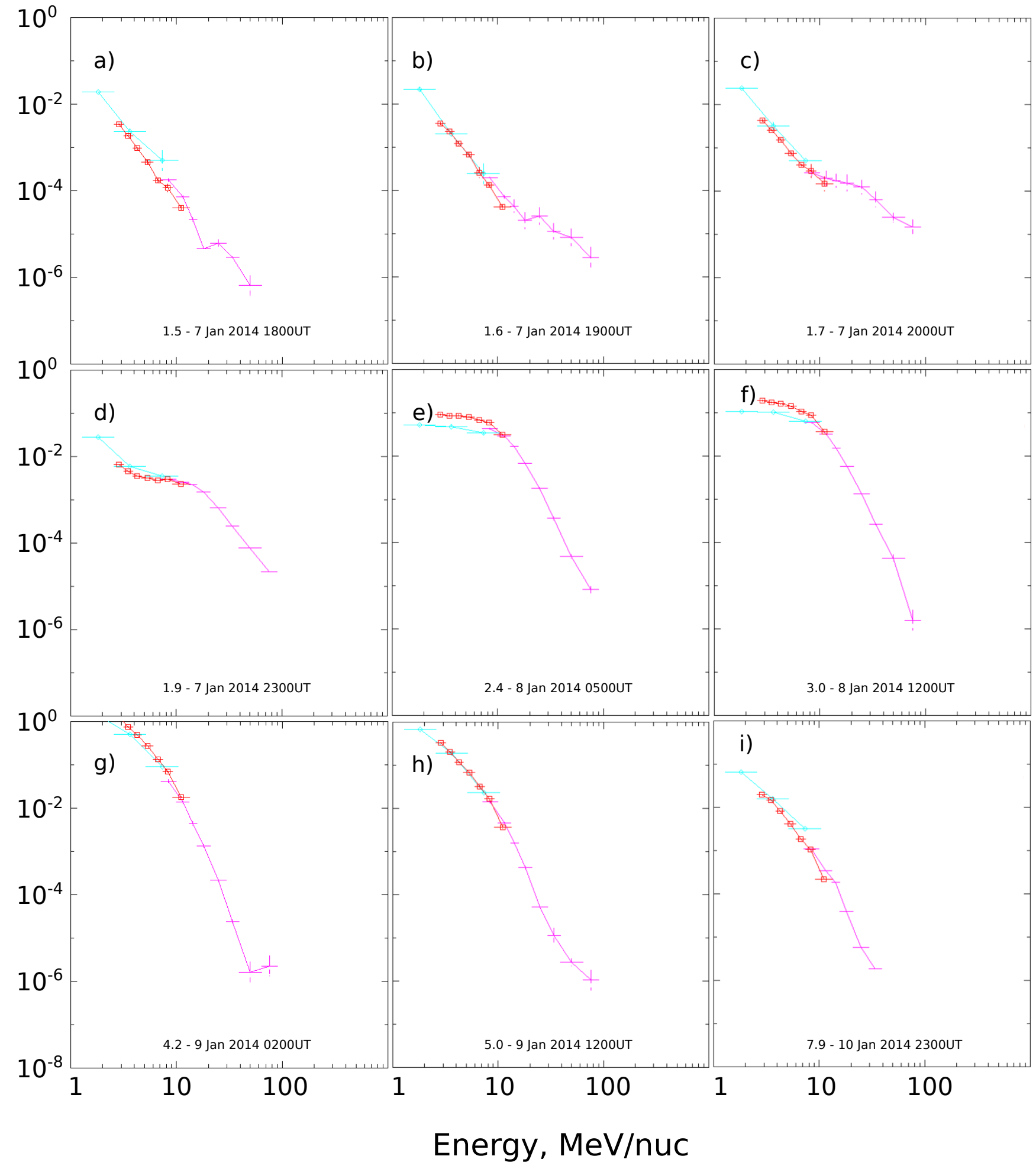

Figure 14. 7 January 2014 event temporal evolution of the oxygen energy spectra for near Earth-located spacecraft ACE/SIS in pink, ACE/ULEIS in light blue, and Wind/EPACT in red, where the numbered panels a) to i) refer to the numbered segments of 12-hour intervals in Figure 13. 
D.J. Doran et al.

1

2

3

4

5

6

7

8

9

10

11

12

13

14

15

16

17

18

19

20

21

22

23

24

25

26

27

28

29

30

31

32

33

34

35

36

37

38

39

40

41

42

43

44

45

46

47

48

49

50

51

52

53

54

55

56

57

58

59

60

61

62

63

64

65

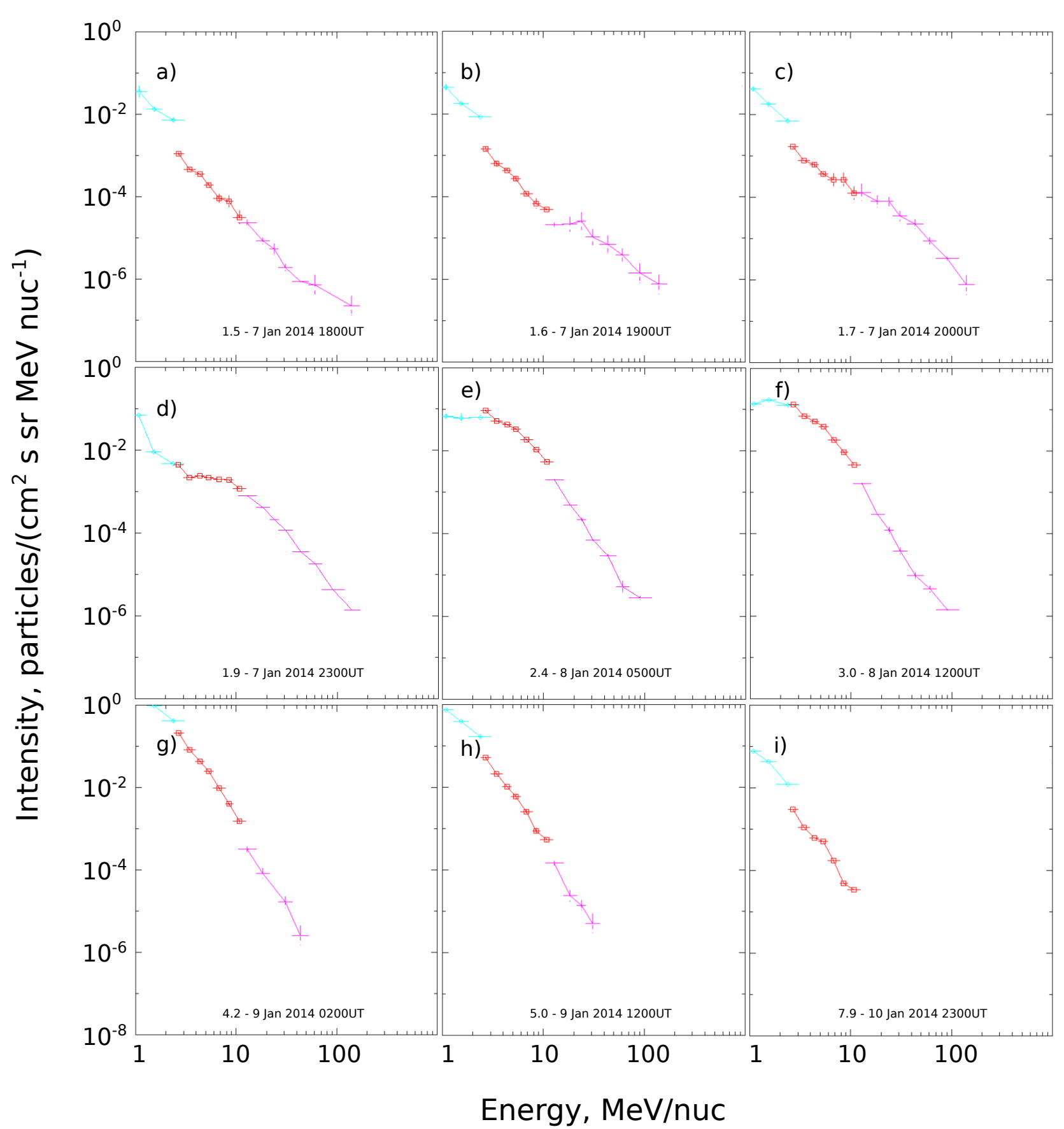

Figure 15. 7 January 2014 event temporal evolution of the iron energy spectra for near Earth-located spacecraft ACE/SIS in pink, ACE/ULEIS in light blue, and Wind/EPACT in red, where the numbered panels a) to i) refer to the numbered segments of 12-hour intervals in Figure 13 


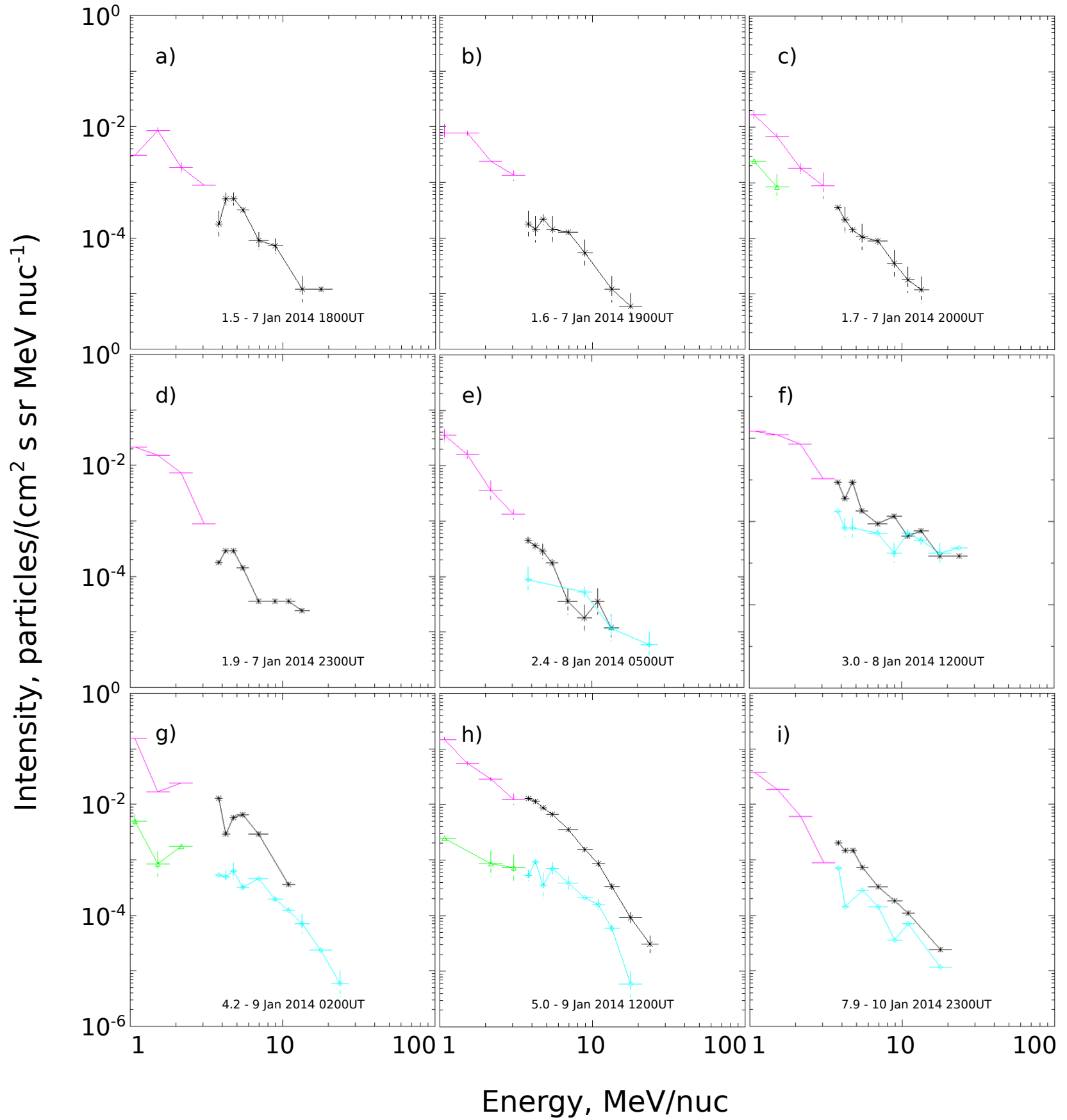

Figure 16. 7 January 2014 event temporal evolution of the oxygen energy spectra for spacecraft STEREO-A and -B, where STEREO-A/LET is in black, STEREO-A/SIT in pink, STEREO-B/LET in light blue, and STEREO-B/SIT in green. The numbered panels a) to i) refer to the numbered segments of 12-hour intervals in Figure 13. 
D.J. Doran et al.

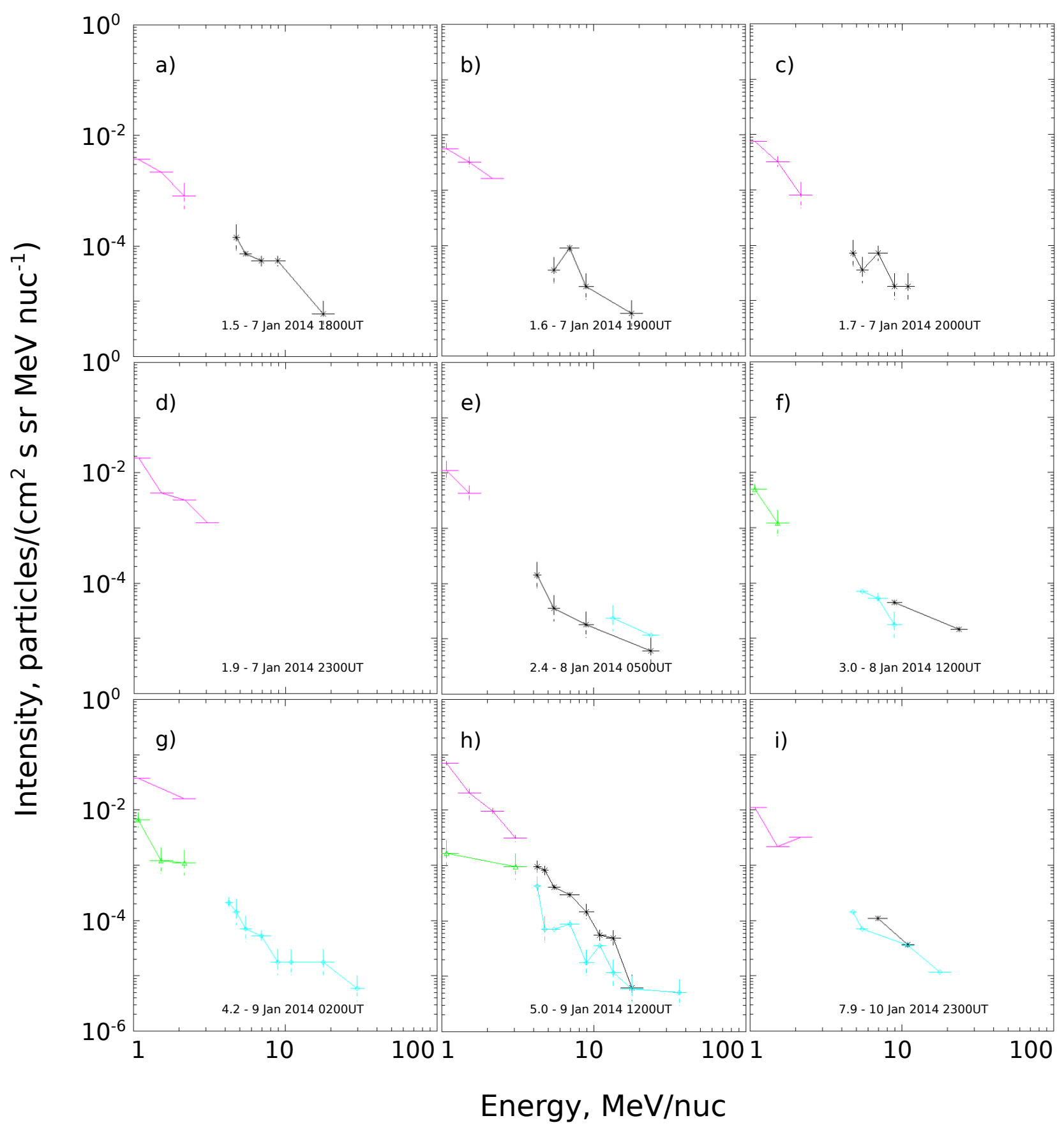

Figure 17. 7 January 2014 event temporal evolution of the iron energy spectra for spacecraft STEREO-A and -B, where STEREO-A/LET is in black, STEREO-A/SIT in pink, STERE$\mathrm{O}-\mathrm{B} / \mathrm{LET}$ in light blue, and STEREO-B/SIT in green. The numbered panels a) to i) refer to the numbered segments of 12-hour intervals in Figure 13. 


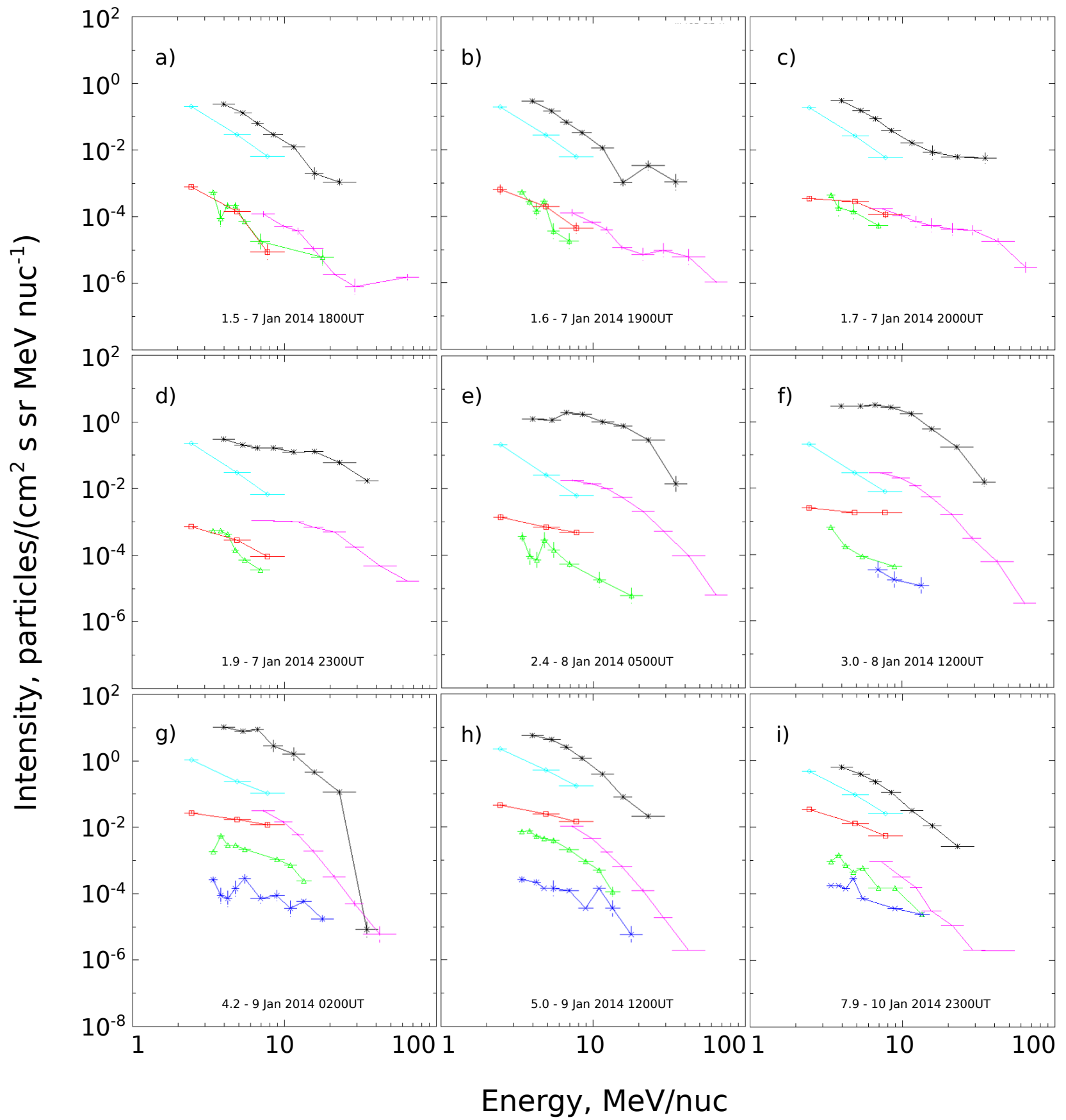

Figure 18. 7 January 2014 event temporal evolution of the helium and carbon spectra for three spacecraft, ACE/SIS, and STEREO-A and -B, where ACE/SIS helium is in black, ACE/SIS carbon in pink, STEREO-A/LET helium in light blue, STEREO-A/LET carbon in green, STEREO-B/LET helium in red, and STEREO-B/LET carbon in blue. The numbered panels a) to i) refer to the numbered segments of 12-hour intervals in Figure 13. 
D.J. Doran et al.

1

2

3

4

5

6

7

8

9

10

11

12

13

14

15

16

17

18

19

20

21

22

23

24

25

26

27

28

29

30

31

32

33

34

35

36

37

38

39

40

41

42

43

44

45

46

47

48

49

50

51

52

53

54

55

56

57

58

59

60

61

62

63

64

65

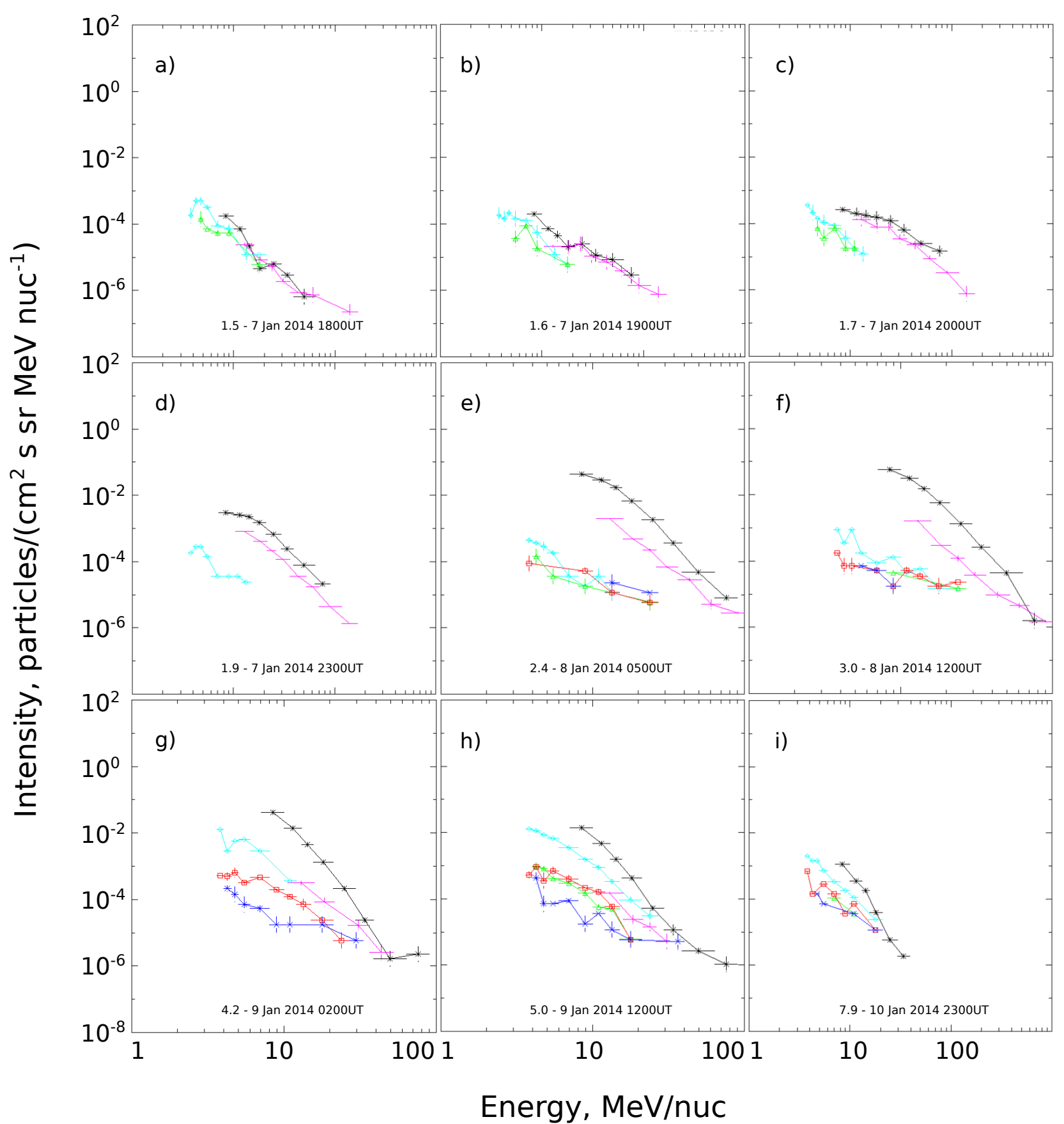

Figure 19. 7 January 2014 event temporal evolution of the oxygen and iron spectra for three spacecraft, ACE/SIS, and STEREO-A and -B, where ACE/SIS oxygen is in black, ACE/SIS iron in pink, STEREO-A/LET oxygen in light blue, STEREO-A/LET iron in green, STEREO-B/LET oxygen in red, and STEREO-B/LET iron in blue. The numbered panels a) to i) refer to the numbered segments of 12-hour intervals in Figure 13. 


\section{References}

Aarnio, A.N., Stassun, K.G., Hughes, W.J., McGregor, S.L.: 2011, Solar Flares and Coronal Mass Ejections: A Statistically Determined Flare Flux-CME Mass Correlation. Solar Phys. 268, 195. DOI. ADS.

Cane, H.V., Reames, D.V., von Rosenvinge, T.T.: 1988, The Role of Interplanetary Shocks in the Longitude Distribution of Solar Energetic Particles. J. Geophys. Res. 93, 9555. DOI. ADS.

Cliver, E.W.: 2009, A Revised Classification Scheme for Solar Energetic Particle Events. Central European Astrophysical Bulletin 33, 253. ADS.

Cohen, C.M.S., Mason, G.M., Stone, E.C., Mewaldt, R.A., Leske, R.A., Desai, M.I., Cummings, A.C., von Rosenvinge, T.T., Wiedenbeck, M.E.: 2005, Solar Energetic Particle Spectra and Composition in the October/November 2003 Events. In: Acharya, B.S., Gupta, S., Jagadeesan, P., Jain, A., Karthikeyan, S., Morris, S., Tonwar, S. (eds.) Internat. Cosmic Ray Conf., Tata Institute of Fundamental Research, Mumbai, 311. ADS.

Compagnino, A., Romano, P., Zuccarello, F.: 2017, A Statistical Study of CME Properties and of the Correlation Between Flares and CMEs over Solar Cycles 23 and 24. Solar Phys. 292, 5. DOI. ADS.

Correia, E., Makhmutov, V., Raulin, J.P., Makita, K.: 2013, Mid- and Low-latitude Response of the Ionosphere to Solar Proton Events on January 2012. J. Phys. CS-409, 012186. DOI. ADS.

Crosby, N., Heynderickx, D., Jiggens, P., Aran, A., Sanahuja, B., Truscott, P., Lei, F., Jacobs, C., Poedts, S., Gabriel, S., Sandberg, I., Glover, A., Hilgers, A.: 2015, SEPEM: A Tool for Statistical Modeling the Solar Energetic Particle Environment. Space Weather 13, 406. DOI.

Dalla, S., Agueda, N.: 2010, Role of Latitude of Source Region in Solar Energetic Particle Events. AIP CP-1216, 613. DOI. ADS.

Dalla, S., Marsh, M.S., Battarbee, M.: 2017, Solar Energetic Particle Drifts and the Energy Dependence of 1 AU Charge States. Astrophys. J. 834, 167. DOI. ADS.

Dalla, S., Marsh, M.S., Laitinen, T.: 2015, Drift-induced Deceleration of Solar Energetic Particles. Astrophys. J. 808, 62. DOI. ADS.

Dalla, S., Balogh, A., Krucker, S., Posner, A., Müller-Mellin, R., Anglin, J.D., Hofer, M.Y., Marsden, R.G., Sanderson, T.R., Heber, B., Zhang, M., McKibben, R.B.: 2003, Characterization of SEP Events at High Heliographic Latitudes. AIP CP-679, 656. DOI. ADS.

Desai, M.I., Mason, G.M., Gold, R.E., Krimigis, S.M., Cohen, C.M.S., Mewaldt, R.A., Mazur, J.E., Dwyer, J.R.: 2006, Heavy-Ion Elemental Abundances in Large Solar Energetic Particle Events and Their Implications for the Seed Population. Astrophys. J. 649, 470. DOI. ADS.

Desai, M.I., Mason, G.M., Dayeh, M.A., Ebert, R.W., Mccomas, D.J., Li, G., Cohen, C.M.S., Mewaldt, R.A., Schwadron, N.A., Smith, C.W.: 2016, Spectral Properties of Large Gradual Solar Energetic Particle Events. I. Fe, O, and Seed Material. Astrophys. J. 816, 68. DOI. ADS.

Doran, D.J., Dalla, S.: 2016, Temporal Evolution of Solar Energetic Particle Spectra. Solar Phys. 291, 2071. DOI. ADS.

Frahm, R.A., Howard, T., DeForest, C., Odstrcil, D., Kallio, E., Mckenna-Lawler, S., Barabash, S., Winningham, J.D., Sharber, J.R., Elliott, H.A.: 2013, Plasma Characteristic Determination During the Coronal Mass Ejection Associated with the January 27, 2012 Solar Storm. In: EGU Gen. Assemb. Conf. Abst., 14062. ADS.

Gómez-Herrero, R., Dresing, N., Klassen, A., Heber, B., Lario, D., Agueda, N., Malandraki, O.E., Blanco, J.J., Rodríguez-Pacheco, J., Banjac, S.: 2015, Circumsolar Energetic Particle Distribution on 2011 November 3. Astrophys. J. 799, 55. DOI. ADS.

Gopalswamy, N.: 2012, Energetic Particle and Other Space Weather Events of Solar Cycle 24. AIP CP-1500, 14. DOI. ADS.

Joshi, N.C., Uddin, W., Srivastava, A.K., Chandra, R., Gopalswamy, N., Manoharan, P.K., Aschwanden, M.J., Choudhary, D.P., Jain, R., Nitta, N.V., Xie, H., Yashiro, S., Akiyama, S., Mäkelä, P., Kayshap, P., Awasthi, A.K., Dwivedi, V.C., Mahalakshmi, K.: 2013, A Multiwavelength Study of Eruptive Events on January 23, 2012 Associated with a Major Solar Energetic Particle Event. Adv. Space Res. 52, 1. DOI. ADS.

Kallenrode, M.-B.: 2004, Space Physics: An Introduction to Plasmas and Particles in the Heliosphere and Magnetospheres, Springer, Berlin. ADS. 
Kallenrode, M.-B., Wibberenz, G., Kunow, H., Müller-Mellin, R., Stolpovskii, V., Kontor, N.: 1993, Multi-spacecraft Observations of Particle Events and Interplanetary Shocks during November/December 1982. Solar Phys. 147, 377. DOI. ADS.

Kóta, J.: 1979, Drift - the Essential Process in Losing Energy. In: Miyake, S. (ed.) Internat. Cosmic Ray Conf. 3, The Institute for Cosmic Ray Research, University of Tokyo, Tokyo, 13. ADS.

Laitinen, T., Kopp, A., Effenberger, F., Dalla, S., Marsh, M.S.: 2016, Solar Energetic Particle Access to Distant Longitudes through Turbulent Field-line Meandering. Astron. Astrophys. 591, A18. DOI. ADS.

Lario, D., Roelof, E.C., Decker, R.B.: 2014, Longitudinal Dependence of SEP Peak Intensities as Evidence of CME-Driven Shock Particle Acceleration. In: Hu, Q., Zank, G.P. (eds.) Outstanding Problems in Heliophysics: From Coronal Heating to the Edge of the Heliosphere CS-484, Astron. Soc. Pacific, San Francisco, 98. ADS.

Lario, D., Aran, A., Gómez-Herrero, R., Dresing, N., Heber, B., Ho, G.C., Decker, R.B., Roelof, E.C.: 2013, Longitudinal and Radial Dependence of Solar Energetic Particle Peak Intensities: STEREO, ACE, SOHO, GOES, and MESSENGER Observations. Astrophys. J. 767, 41. DOI. ADS.

Lario, D., Raouafi, N.E., Kwon, R.-Y., Zhang, J., Gómez-Herrero, R., Dresing, N., Riley, P.: 2014, The Solar Energetic Particle Event on 2013 April 11: An Investigation of its Solar Origin and Longitudinal Spread. Astrophys. J. 797, 8. DOI. ADS.

Li, G., Zank, G.P., Verkhoglyadova, O., Mewaldt, R.A., Cohen, C.M.S., Mason, G.M., Desai, M.I.: 2009, Shock Geometry and Spectral Breaks in Large SEP Events. Astrophys. J. 702, 998. DOI. ADS.

Martirosyan, H.S.: 2005, Classification of Solar Events. I. Classification According to Knee Energy in Energy Ion Spectra. Astrophysi. 48, 208. DOI. ADS.

Mason, G.M., Mazur, J.E., Dwyer, J.R.: 2002, A New Heavy Ion Abundance Enrichment Pattern in ${ }^{3}$ He-rich Solar Particle Events. Astrophys. J. Lett. 565, L51. DOI. ADS.

Mays, M.L., Thompson, B.J., Jian, L.K., Colaninno, R.C., Odstrcil, D., Möstl, C., Temmer, M., Savani, N.P., Collinson, G., Taktakishvili, A., MacNeice, P.J., Zheng, Y.: 2015, Propagation of the 7 January 2014 CME and Resulting Geomagnetic Non-event. Astrophys. J. 812, 145. DOI. ADS.

Mewaldt, R.A., Looper, M.D., Cohen, C.M.S., Haggerty, D.K., Labrador, A.W., Leske, R.A., Mason, G.M., Mazur, J.E., von Rosenvinge, T.T.: 2012, Energy Spectra, Composition, and Other Properties of Ground-Level Events During Solar Cycle 23. Space Sci. Rev. 171, 97. DOI. ADS.

Park, J., Innes, D.E., Bucik, R., Moon, Y.-J.: 2013, The Source Regions of Solar Energetic Particles Detected by Widely Separated Spacecraft. Astrophys. J. 779, 184. DOI. ADS.

Parker, E.N.: 1965, The Passage of Energetic Charged Particles through Interplanetary Space. Planet. Space Sci. 13, 9. DOI. ADS.

Reames, D.V.: 1995, Solar Energetic Particles: A Paradigm Shift. Rev. Geophys. 33, 585. DOI. ADS.

Reames, D.V.: 1998, Solar Energetic Particles: Sampling Coronal Abundances. Space Sci. Rev. 85, 327. DOI. ADS.

Reames, D.V.: 1999, Particle Acceleration at the Sun and in the Heliosphere. Space Sci. Rev. 90, 413. DOI. ADS.

Reames, D.V.: 2014, Element Abundances in Solar Energetic Particles and the Solar Corona. Solar Phys. 289, 977. DOI. ADS.

Reames, D.V., Ng, C.K.: 2004, Heavy-Element Abundances in Solar Energetic Particle Events. Astrophys. J. 610, 510. DOI. ADS.

Reames, D.V., Barbier, L.M., Ng, C.K.: 1996, The Spatial Distribution of Particles Accelerated by Coronal Mass Ejection-driven Shocks. Astrophys. J. 466, 473. DOI. ADS.

Reames, D.V., Ng, C.K., Tylka, A.J.: 2001, Heavy Ion Abundances and Spectra and the Large Gradual Solar Energetic Particle Event of 2000 July 14. Astrophys. J. Lett. 548, L233. DOI. ADS.

Reames, D.V., Ng, C.K., Tylka, A.J.: 2013, Spatial Distribution of Solar Energetic Particles in the Inner Heliosphere. Solar Phys. 285, 233. DOI. ADS.

Reames, D.V., Barbier, L.M., Von Rosenvinge, T. T., Mason, G.M., Mazur, J.E., Dwyer, J. R.: 1997, Energy Spectra of Ions Accelerated in Impulsive and Gradual Solar Events. Astrophys. J. 483, 515. ADS.

Richardson, I.G., von Rosenvinge, T.T., Cane, H.V., Christian, E.R., Cohen, C.M.S., Labrador, A.W., Leske, R.A., Mewaldt, R.A., Wiedenbeck, M.E., Stone, E.C.: 2014, $>25 \mathrm{MeV}$ proton 
events observed by the High Energy Telescopes on the STEREO A and B spacecraft and/or at Earth during the first seven years of the STEREO mission. Solar Phys. 289, 3059. DOI.

Rodríguez-Gasén, R., Aran, A., Sanahuja, B., Jacobs, C., Poedts, S.: 2014, Variation of Proton Flux Profiles with the Observer's Latitude in Simulated Gradual SEP Events. Solar Phys. 289, 1745. DOI. ADS

Ruffolo, D.: 1995, Effect of adiabatic deceleration on the focused transport of solar cosmic rays. Astrophys. J. 442, 861. DOI. ADS.

Shanmugaraju, A., Moon, Y.-J., Vršnak, B.: 2011, Correlation between CME and Flare Parameters (with and without Type II Bursts). Solar Phys. 270, 273. DOI. ADS.

Thakur, N., Gopalswamy, N., Xie, H., Mäkelä, P., Yashiro, S., Akiyama, S., Davila, J.M.: 2014, Ground Level Enhancement in the 2014 January 6 Solar Energetic Particle Event. Astrophys. J. Lett. 790, L13. DOI. ADS.

Tylka, A.J., Lee, M.A.: 2006, A Model for Spectral and Compositional Variability at High Energies in Large, Gradual Solar Particle Events. Astrophys. J. 646, 1319. DOI. ADS.

Vainio, R., Valtonen, E., Heber, B., Malandraki, O.E., Papaioannou, A., Klein, K.-L., Afanasiev, A., Agueda, N., Aurass, H., Battarbee, M., Braune, S., Dröge, W., Ganse, U., Hamadache, C., Heynderickx, D., Huttunen-Heikinmaa, K., Kiener, J., Kilian, P., Kopp, A., Kouloumvakos, A., Maisala, S., Mishev, A., Miteva, R., Nindos, A., Oittinen, T., Raukunen, O., Riihonen, E., Rodríguez-Gasén, R., Saloniemi, O., Sanahuja, B., Scherer, R., Spanier, F., Tatischeff, V., Tziotziou, K., Usoskin, I.G., Vilmer, N.: 2013, The first SEPServer event catalogue $68-\mathrm{MeV}$ solar proton events observed at 1 AU in 1996-2010. Space Weather Space Climate 3(27), A12. DOI. ADS.

Vršnak, B.: 2016, Solar eruptions: The CME-flare relationship. Astron. Nach. 337, 1002. DOI. ADS.

Watanabe, K., Shimizu, T., Masuda, S., Ichimoto, K., Ohno, M.: 2013, Emission Height and Temperature Distribution of White-light Emission Observed by Hinode/SOT from the 2012 January 27 X-class Solar Flare. Astrophys. J. 776, 123. DOI. ADS.

Wibberenz, G., Cane, H.V.: 1993, Multi-Spacecraft Observations of Particles and Shocks in the Inner Heliosphere. In: Leahy, D.A., Hicks, R.B., Venkatesan, D. (eds.) Internat. Cosmic Ray Conf. 3, World Scientific, Calgary, 274. ADS.

Wiedenbeck, M.E., Mason, G.M., Cohen, C.M.S., Nitta, N.V., Gómez-Herrero, R., Haggerty, D.K.: 2013, Observations of Solar Energetic Particles from ${ }^{3}$ He-rich Events over a Wide Range of Heliographic Longitude. Astrophys. J. 762, 54. DOI. ADS.

Zelina, P., Dalla, S., Cohen, C.M.S., Mewaldt, R.A.: 2017, Time Evolution of Elemental Ratios in Solar Energetic Particle Events. Astrophys. J. 835, 71. DOI. ADS 